\title{
Diffuse measures and nonlinear parabolic equations
}

\author{
Francesco Petitta, Augusto C. Ponce and Alessio Porretta
}

Abstract. Given a parabolic cylinder $Q=(0, T) \times \Omega$, where $\Omega \subset \mathbb{R}^{N}$ is a bounded domain, we prove new properties of solutions of

$$
u_{t}-\Delta_{p} u=\mu \text { in } Q
$$

with Dirichlet boundary conditions, where $\mu$ is a finite Radon measure in $Q$. We first prove a priori estimates on the $p$-parabolic capacity of level sets of $u$. We then show that diffuse measures (i.e., measures which do not charge sets of zero parabolic $p$-capacity) can be strongly approximated by the measures $\mu_{k}=\left(T_{k}(u)\right)_{t}-\Delta_{p}\left(T_{k}(u)\right)$, and we introduce a new notion of renormalized solution based on this property. We finally apply our new approach to prove the existence of solutions of

$$
u_{t}-\Delta_{p} u+h(u)=\mu \text { in } Q
$$

for any function $h$ such that $h(s) s \geq 0$ and for any diffuse measure $\mu$; when $h$ is nondecreasing, we also prove uniqueness in the renormalized formulation. Extensions are given to the case of more general nonlinear operators in divergence form.

\section{Introduction and main results}

Given a bounded domain $\Omega \subset \mathbb{R}^{N}$ and $T>0$, let $Q=(0, T) \times \Omega$. We denote by $\mathcal{M}(Q)$ the vector space of all finite Radon measures in $Q$ equipped with the norm $\|\mu\|_{\mathcal{M}(Q)}=|\mu|(Q)$. This paper is motivated by the study of the evolution problem

$$
\begin{cases}u_{t}-\Delta_{p} u+h(u)=\mu & \text { in } Q, \\ u=u_{0} & \text { on }\{0\} \times \Omega, \\ u=0 & \text { on }(0, T) \times \partial \Omega,\end{cases}
$$

where $-\Delta_{p} u=-\operatorname{div}\left(|\nabla u|^{p-2} \nabla u\right)$ is the $p$-Laplace operator, $p>1, \mu \in$ $\mathcal{M}(Q), u_{0} \in L^{1}(\Omega)$, and $h: \mathbb{R} \rightarrow \mathbb{R}$ is a continuous function such that $h(s) s \geq 0$ for large $|s|$. It is well known (see e.g., [1]) that problem (1.1) may not have a solution for every measure $\mu$ (unless some growth restriction is imposed on $h$ ). As suggested

Keywords: Parabolic capacity, measure data, nonlinear equations with absorption, renormalized solutions.

The first author is partially supported by the Spanish PNPGC project, reference MTM2008-03176. The second author (A.C.P.) was partially supported by the Fonds de la Recherche scientifique-FNRS (Belgium) and by the Fonds spéciaux de Recherche (Université catholique de Louvain). 
by the stationary case (see [9]), if one looks for a "general solvability" result (i.e., existence of solutions of (1.1) for any function $h$ ), then it is necessary to restrict the attention to the class of measures $\mu$ which do not charge sets of zero capacity. Here, the notion of capacity which is relevant is the so-called parabolic p-capacity.

To be precise, we recall that for every $p>1$ and every open subset $U \subset Q$, the $p$-parabolic capacity of $U$ is given by (see $[13,21]$ )

$$
\operatorname{cap}_{p}(U)=\inf \left\{\|u\|_{W}: u \in W, u \geq \chi_{U} \text { a.e. in } Q\right\}
$$

where

$$
W=\left\{u \in L^{p}(0, T ; V): u_{t} \in L^{p^{\prime}}\left(0, T ; V^{\prime}\right)\right\}
$$

being $V=W_{0}^{1, p}(\Omega) \cap L^{2}(\Omega)$ and $V^{\prime}$ its dual space. As usual, $W$ is endowed with the norm

$$
\|u\|_{W}=\|u\|_{L^{p}(0, T ; V)}+\left\|u_{t}\right\|_{L^{p^{\prime}\left(0, T ; V^{\prime}\right)}} \cdot
$$

The $p$-parabolic capacity cap $p$ is then extended to arbitrary Borel subsets $B \subset Q$ as

$$
\operatorname{cap}_{p}(B)=\inf \left\{\operatorname{cap}_{p}(U): B \subset U \text { and } U \subset Q \text { is open }\right\} .
$$

Henceforth, we call a finite measure $\mu$ diffuse if it does not charge sets of zero $p$ parabolic capacity, i.e., if $\mu(E)=0$ for every Borel set $E \subset Q \operatorname{such}$ that $\operatorname{cap}_{p}(E)=0$. The subspace of all diffuse measures in $Q$ will be denoted by $\mathcal{M}_{0}(Q)$.

One of our goals is to prove that (1.1) admits a solution for every diffuse measure and every $h$ satisfying the sign condition. In the elliptic case, such result can be proved using the representation of diffuse measures as elements in $L^{1}(\Omega)+W^{-1, p^{\prime}}(\Omega)$ (see [8]). Moreover, if $h$ is increasing, then (entropy or renormalized) solutions are unique.

In the parabolic case, the situation is more delicate. According to a representation theorem for diffuse measures proved in [13], for every $\mu \in \mathcal{M}_{0}(Q)$, there exist $f \in L^{1}(Q), g \in L^{p}(0, T ; V)$ and $\chi \in L^{p^{\prime}}\left(0, T ; W^{-1, p^{\prime}}(\Omega)\right)$ such that

$$
\mu=f+g_{t}+\chi \quad \text { in } \mathcal{D}^{\prime}(Q)
$$

In the same paper, in order to deal with nonlinear equations where the source term is a diffuse measure, the authors introduced a renormalized formulation that is based on this representation. However, in contrast with the elliptic case, such representation, as well as the formulation suggested in [13], is not suitable to handle the case of absorption terms as in (1.1). The main reason is that a solution of

$$
u_{t}-\Delta_{p} u=\mu=f+\chi+g_{t} \text { in } Q
$$

is meant in the sense that $v=u-g$ satisfies

$$
v_{t}-\Delta_{p}(v+g)=f+\chi \quad \text { in } Q .
$$


The same approach for problem (1.1) would transform the absorption term $h(u)$ into $h(v+g)$. However, since no growth restriction is made on $h$, this term can not be easily handled if $g$ is not bounded.

Since the decomposition (1.5) is not uniquely determined, a natural question would be whether every diffuse measure can be written as (1.5) for some $g \in L^{\infty}(Q)$. Unfortunately, the answer is no, as we show in Example 3.1.

In this paper, we overcome this obstruction by developing a different approach to deal with diffuse measures. In this way, we establish new properties of diffuse measures (related to different types of approximations) and new results for parabolic equations (including (1.1)). Both issues are closely related as we will see later.

As far as diffuse measures are concerned, one of the results that we prove is that every $\mu \in \mathcal{M}_{0}(Q)$ can be strongly approximated by measures which admit decomposition (1.5) with $g \in L^{\infty}(Q)$.

THEOREM 1.1. Let $\mu \in \mathcal{M}_{0}(Q)$. Then, for every $\varepsilon>0$ there exists $v \in \mathcal{M}_{0}(Q)$ such that

$$
\|\mu-v\|_{\mathcal{M}(Q)} \leq \varepsilon \text { and } \quad v=w_{t}-\Delta_{p} w \text { in } \mathcal{D}^{\prime}(Q)
$$

where $w \in L^{p}\left(0, T ; W_{0}^{1, p}(\Omega)\right) \cap L^{\infty}(Q)$.

Not only is this density result interesting in itself, but also the construction and the properties of the approximation $v$ are important. Indeed, the function $w$ is constructed as the truncation of a nonlinear potential of $\mu$ (we call in this way a function $u$ such that $u_{t}-\Delta_{p} u=\mu$ ). As a consequence, the approximation property for the measure $\mu$ is linked to a property of its nonlinear potential.

The main ingredient in the proof of Theorem 1.1 is a capacitary estimate on the level sets of $u$. Such estimates for solutions of parabolic equations have an independent interest and read as follows.

THEOREM 1.2. Given $\mu \in \mathcal{M}(Q) \cap L^{p^{\prime}}\left(0, T ; W^{-1, p^{\prime}}(\Omega)\right)$ and $u_{0} \in L^{2}(\Omega)$, let $u \in W$ be the (unique) weak solution of

$$
\begin{cases}u_{t}-\Delta_{p} u=\mu & \text { in } Q, \\ u=u_{0} & \text { on }\{0\} \times \Omega, \\ u=0 & \text { on }(0, T) \times \partial \Omega .\end{cases}
$$

Then,

$$
\operatorname{cap}_{p}(\{|u|>k\}) \leq C \max \left\{\frac{1}{k^{\frac{1}{p}}}, \frac{1}{k^{\frac{1}{p^{\prime}}}}\right\} \quad \forall k \geq 1,
$$

where $C>0$ is a constant depending on $\|\mu\|_{\mathcal{M}(Q)},\left\|u_{0}\right\|_{L^{1}(\Omega)}$, and $p$.

In (1.8), we have identified $u$ with its cap-quasi continuous representative, which exists since $u \in W$ (see [13]). In particular, the quantity $\operatorname{cap}_{p}(\{|u|>k\})$ is welldefined. 
Given a diffuse measure $\mu$, we apply Theorem 1.2 to construct a measurable function $u: Q \rightarrow \mathbb{R}$ such that the truncations $T_{k}(u)$ satisfy

$$
\left(T_{k}(u)\right)_{t}-\Delta_{p}\left(T_{k}(u)\right)=\mu+\lambda_{k} \text { in } Q
$$

for a sequence of measures $\left(\lambda_{k}\right)$ such that

$$
\left\|\lambda_{k}\right\|_{\mathcal{M}(Q)} \rightarrow 0
$$

We deduce in particular the strong approximation property given in Theorem 1.1. On the other hand, this result also motivates an alternative formulation of the concept of renormalized solution of

$$
\begin{cases}u_{t}-\Delta_{p} u=\mu & \text { in } Q, \\ u=u_{0} & \text { on }\{0\} \times \Omega, \\ u=0 & \text { on }(0, T) \times \partial \Omega .\end{cases}
$$

in terms of properties (1.9)-(1.10). Such a formulation, no more based on the decomposition (1.5), can be extended to problem (1.1) straightforwardly and turns out to be suitable to tackle the absorption problem.

We prove that this formulation extends the one given in [4] for $L^{1}$-data and, in case of problem (1.11), is equivalent with the definition for diffuse measures given in [13] (and therefore equivalent with the entropic formulation in [14]). This formulation we use is largely inspired by (and it is very close to) other versions of renormalized formulations in the literature as, for example, in [12] for elliptic equations and [3] for conservation laws.

We obtain in this way a new approach to solve nonlinear problems involving diffuse measures:

THEOREM 1.3. Let $\mu \in \mathcal{M}_{0}(Q)$ and $u_{0} \in L^{1}(\Omega)$. Let $h: \mathbb{R} \rightarrow \mathbb{R}$ be a continuous function satisfying

$$
h(s) s \geq 0 \text { for every }|s|>L,
$$

for some $L \geq 0$. Then, (1.1) admits a renormalized solution (which is, in particular, a distributional solution). If in addition $h$ is nondecreasing, then the renormalized solution is unique.

The proof of Theorem 1.3 strongly relies on the new ingredients developed so far. The existence of a solution $u$ is obtained as limit of solutions $u_{n}$ corresponding to a smooth approximation $\mu_{n}$ of the measure $\mu$. In this procedure, the difficult point is to prove the $L^{1}$-convergence of the lower order term $h\left(u_{n}\right)$. When the sequence $\left(\mu_{n}\right)$ is strongly (or even weakly) converging in $L^{1}(Q)$, this is usually deduced (see e.g., [16]) from the estimate

$$
\int_{\left\{\left|u_{n}\right|>k\right\}}\left|h\left(u_{n}\right)\right| \leq \int_{\left\{\left|u_{n}\right|>k\right\}}\left|\mu_{n}\right|,
$$


using the equi-integrability of $\left(\mu_{n}\right)$. In our case, we extend this idea in the following sense: the level sets $\left\{\left|u_{n}\right|>k\right\}$ are proved to have uniformly small capacity (Theorem 1.2) and the sequence $\left(\mu_{n}\right)$ is chosen to be equidiffuse (see Definition 3.2 below), a concept introduced in [10]; see also [18]. An example of such sequence is given by the convolution $\mu_{n}=\rho_{n} * \mu$, where the measure $\mu$ is diffuse (see Proposition 3.3 below). Equidiffuse sequences play the same role for the capacity, as do equi-integrable sequences for the Lebesgue measure. Therefore, coupling the capacitary estimates with the equidiffuse property of $\left(\mu_{n}\right)$, the right-hand side of (1.12) is uniformly small, implying the $L^{1}$-convergence of $\left(h\left(u_{n}\right)\right)$. Finally, when $h$ is nondecreasing, we obtain uniqueness by proving that the $L^{1}$-contraction property holds for renormalized solutions.

The article is organized in the following way. In Sect. 2, we prove Theorem 1.2. In Sect. 3, we discuss some properties of diffuse measures related to the representation (1.5) and we prove Theorem 1.1. In Sect. 4, we discuss our new renormalized formulation of problem (1.11): definition, existence and uniqueness, and some properties of renormalized solutions, including a generalized version of the capacitary estimates of Theorem 1.2 (see Proposition 4.8). In Sect. 5, we turn our attention to problem (1.1) and we prove Theorem 1.3. These results will be actually proved in the context of nonlinear monotone operators in divergence form. We will briefly sketch in Sect. 6 some extension to the case of nonmonotone operators.

Part of the results of Sects. 2 and 3 was announced (and proved for the case of positive measures) in [20]; nevertheless, we include here all the details and give a self contained exposition for the sake of clarity.

\section{Capacitary estimates: proof of Theorem 1.2}

Let us first recall the following fact that will be used later. A set $E \subset Q$ is called cap-quasi open if for every $\varepsilon>0$ there exists an open set $A_{\varepsilon} \subset Q$ such that $E \subset A_{\varepsilon}$ and $\operatorname{cap}_{p}\left(A_{\varepsilon} \backslash E\right)<\varepsilon$. The interest in such sets arises since, if a function $z$ has a cap-quasi continuous representative, then its level sets $\{(x, t) \in Q: z(x, t)>a\}$ are cap-quasi open. It is well known that the capacity of cap-quasi open sets can be estimated as follows

LEMMA 2.1. Let E be a cap-quasi open subset in $Q$. Then

$$
\operatorname{cap}_{p}(E) \leq \inf \left\{\|v\|_{W}: v \in W, v \geq \chi_{E} \text { a.e. in } Q\right\}
$$

Proof. Let $\varepsilon>0$ and $A_{\varepsilon}$ be an open set such that $E \subset A_{\varepsilon}$ and $\operatorname{cap}_{p}\left(A_{\varepsilon} \backslash E\right)<\varepsilon$. By definition of capacity, there exists another open set $U_{\varepsilon}$ such that $A_{\varepsilon} \backslash E \subset U_{\varepsilon}$ and $\operatorname{cap}_{p}\left(U_{\varepsilon}\right)<\varepsilon$. Then, let $w_{\varepsilon} \in W$ such that $w_{\varepsilon} \geq \chi_{U_{\varepsilon}}$ a.e. and $\left\|w_{\varepsilon}\right\|_{W} \leq \varepsilon$. Consider the open set $A_{\varepsilon} \cup U_{\varepsilon}$; for any $v \geq \chi_{E}$ a.e., we have $v+w_{\varepsilon} \geq \chi_{U_{\varepsilon} \cup A_{\varepsilon}}$ a.e. in $Q$, hence by (1.2)

$$
\operatorname{cap}_{p}\left(U_{\varepsilon} \cup A_{\varepsilon}\right) \leq\|v\|_{W}+\left\|w_{\varepsilon}\right\|_{W} \leq\|v\|_{W}+\varepsilon .
$$


We deduce that

$$
\operatorname{cap}_{p}(E) \leq \operatorname{cap}_{p}\left(A_{\varepsilon} \cup U_{\varepsilon}\right) \leq\|v\|_{W}+\varepsilon,
$$

and letting $\varepsilon \rightarrow 0$ we get

$$
\operatorname{cap}_{p}(E) \leq\|v\|_{W}
$$

Since $v$ is arbitrary we conclude.

Throughout this paper, we consider a sequence of mollifiers $\left(\rho_{n}\right)$ such that for every $n \geq 1$,

$$
\rho_{n} \in C_{c}^{\infty}\left(\mathbb{R}^{N+1}\right), \quad \operatorname{supp} \rho_{n} \subset B_{\frac{1}{n}}(0), \quad \rho_{n} \geq 0, \quad \text { and } \int_{\mathbb{R}^{N+1}} \rho_{n}=1 .
$$

Given $\mu \in \mathcal{M}(Q)$, we define the convolution $\rho_{n} * \mu$ for every $(t, x) \in \mathbb{R} \times \mathbb{R}^{N}$ by

$$
\left(\rho_{n} * \mu\right)(t, x)=\int_{Q} \rho_{n}(t-s, x-y) \mathrm{d} \mu(s, y) .
$$

Before proving Theorem 1.2, we recall that if $u \in W$, then $u$ is a weak solution of (1.7) if

$$
\int_{0}^{T}\left\langle u_{t}, v\right\rangle \mathrm{d} t+\int_{Q}|\nabla u|^{p-2} \nabla u \nabla v \mathrm{~d} x \mathrm{~d} t=\int_{0}^{T}\langle\mu, v\rangle \mathrm{d} t \quad \forall v \in W,
$$

where $\langle\cdot, \cdot\rangle$ denotes the duality between $V$ and $V^{\prime}$.

If $\mu \in \mathcal{M}(Q) \cap L^{p^{\prime}}\left(0, T ; W^{-1, p^{\prime}}(\Omega)\right)$, then (2.2) holds for every $v \in L^{p}(0, T ; V)$, and actually for every $r \in[0, T]$ we have

$$
\int_{0}^{r}\left\langle u_{t}, v\right\rangle \mathrm{d} t+\int_{0}^{r} \int_{\Omega}|\nabla u|^{p-2} \nabla u \nabla v \mathrm{~d} x \mathrm{~d} t=\int_{0}^{r}\langle\mu, v\rangle \mathrm{d} t,
$$

for every $v \in L^{p}(0, T ; V)$.

A useful identity we shall use is the following: if $u \in W$, then

$$
\int_{s}^{t}\left\langle u_{t}, \psi^{\prime}(u)\right\rangle d t=\int_{\Omega} \psi(u(t)) \mathrm{d} x-\int_{\Omega} \psi(u(s)) \mathrm{d} x,
$$

for every $s, t \in[0, T]$ and every function $\psi: \mathbb{R} \rightarrow \mathbb{R}$ such that $\psi^{\prime}$ is Lipschitz continuous and $\psi^{\prime}(0)=0$. Indeed, since $V=W_{0}^{1, p}(\Omega) \cap L^{2}(\Omega)$, we have $L^{p^{\prime}}\left(0, T ; V^{\prime}\right)=$ $L^{p^{\prime}}\left(0, T ; W^{-1, p^{\prime}}(\Omega)\right)+L^{p^{\prime}}\left(0, T ; L^{2}(\Omega)\right)$. Using the density of $C_{c}^{\infty}([0, T] \times \Omega)$ in $W$ (see e.g., [13, Theorem 2.11]) and the embedding $W \subset C^{0}\left([0, T] ; L^{2}(\Omega)\right)$, one obtains (2.4).

Proof of Theorem 1.2. We define, for any positive $k$,

$$
T_{k}(s)=\max \{-k, \min \{k, s\}\} \quad \forall s \in \mathbb{R} .
$$


We divide the proof into a few steps.

Step 1. Estimates of $T_{k}(u)$ in the space $L^{\infty}\left(0, T ; L^{2}(\Omega)\right) \cap L^{p}\left(0, T ; W_{0}^{1, p}(\Omega)\right)$.

For every $\tau \in \mathbb{R}$, let

$$
\Theta_{k}(\tau)=\int_{0}^{\tau} T_{k}(\sigma) \mathrm{d} \sigma
$$

Take $r \in[0, T]$. Applying (2.3) with $v=T_{k}(u)$ and (2.4) with $\psi=\Theta_{k}, s=0$ and $t=r$, we have

$$
\int_{\Omega} \Theta_{k}(u)(r) \mathrm{d} x+\int_{0}^{r} \int_{\Omega}\left|\nabla T_{k}(u)\right|^{p} \mathrm{~d} x \mathrm{~d} t \leq k\|\mu\|_{\mathcal{M}(Q)}+\int_{\Omega} \Theta_{k}\left(u_{0}\right) \mathrm{d} x,
$$

Observing that $\frac{T_{k}(s)^{2}}{2} \leq \Theta_{k}(s) \leq k|s|, \forall s \in \mathbb{R}$, we have

$$
\int_{\Omega} \frac{\left[T_{k}(u)(r)\right]^{2}}{2} \mathrm{~d} x+\int_{0}^{r} \int_{\Omega}\left|\nabla T_{k}(u)\right|^{p} \mathrm{~d} x \mathrm{~d} t \leq k\left(\|\mu\|_{\mathcal{M}(Q)}+\left\|u_{0}\right\|_{L^{1}(\Omega)}\right)
$$

for any $r \in[0, T]$. In particular, we deduce

$$
\left\|T_{k}(u)\right\|_{L^{\infty}\left(0, T ; L^{2}(\Omega)\right)}^{2} \leq 2 k M \text { and }\left\|T_{k}(u)\right\|_{L^{p}\left(0, T ; W_{0}^{1, p}(\Omega)\right)}^{p} \leq k M,
$$

where

$$
M=\|\mu\|_{\mathcal{M}(Q)}+\left\|u_{0}\right\|_{L^{1}(\Omega)} .
$$

Step 2. Estimates in $W$.

In order to deduce some estimate in $W$, we use an idea from [21]. By standard results (see [17]), there exists a unique solution $z \in L^{\infty}\left(0, T ; L^{2}(\Omega)\right) \cap L^{p}\left(0, T ; W_{0}^{1, p}(\Omega)\right)$ of the backward problem

$$
\begin{cases}-z_{t}-\Delta_{p} z=-2 \Delta_{p} T_{k}(u) & \text { in } Q \\ z=T_{k}(u) & \text { on }\{T\} \times \Omega \\ z=0 & \text { on }(0, T) \times \partial \Omega\end{cases}
$$

Let us multiply (2.8) by $z$ and integrate between $\tau$ and $T$. Using Young's inequality, we obtain

$$
\begin{aligned}
& \int_{\Omega} \frac{[z(\tau)]^{2}}{2} \mathrm{~d} x+\frac{1}{2} \int_{\tau}^{T} \int_{\Omega}|\nabla z|^{p} \mathrm{~d} x \mathrm{~d} t \leq \int_{\Omega} \frac{\left[T_{k}(u)(T)\right]^{2}}{2} \mathrm{~d} x \\
& \quad+C \int_{\tau}^{T} \int_{\Omega}\left|\nabla T_{k}(u)\right|^{p} \mathrm{~d} x \mathrm{~d} t
\end{aligned}
$$

for every $\tau \in[0, T]$. Using (2.5) with $r=T$, we deduce

$$
\int_{\Omega} \frac{[z(\tau)]^{2}}{2} \mathrm{~d} x+\frac{1}{2} \int_{\tau}^{T} \int_{\Omega}|\nabla z|^{p} \mathrm{~d} x \mathrm{~d} t \leq C k\left(\|\mu\|_{\mathcal{M}(Q)}+\left\|u_{0}\right\|_{L^{1}(\Omega)}\right)=C k M
$$


for every $\tau \in[0, T]$. This implies

$$
\|z\|_{L^{\infty}\left(0, T ; L^{2}(\Omega)\right)}^{2}+\|z\|_{L^{p}\left(0, T ; W_{0}^{1, p}(\Omega)\right)}^{p} \leq C k M .
$$

Recall that $V=W_{0}^{1, p}(\Omega) \cap L^{2}(\Omega)$; thus,

$$
\|z\|_{L^{p}(0, T ; V)}^{p} \leq C\left(\|z\|_{L^{p}\left(0, T ; W_{0}^{1, p}(\Omega)\right)}^{p}+\|z\|_{L^{p}\left(0, T ; L^{2}(\Omega)\right)}^{p}\right) .
$$

We deduce from (2.9) that

$$
\|z\|_{L^{p}(0, T ; V)} \leq C\left[(k M)^{\frac{1}{p}}+(k M)^{\frac{1}{2}}\right] .
$$

Moreover, the equation in (2.8) implies

$$
\left\|z_{t}\right\|_{L^{p^{\prime}}\left(0, T ; W^{\left.-1, p^{\prime}(\Omega)\right)}\right.} \leq C\left(\|z\|_{L^{p}\left(0, T ; W_{0}^{1, p}(\Omega)\right)}^{p-1}+\left\|T_{k}(u)\right\|_{L^{p}\left(0, T ; W_{0}^{1, p}(\Omega)\right)}^{p-1}\right) .
$$

Hence, using (2.6) and (2.9), we deduce

$$
\left\|z_{t}\right\|_{L^{p^{\prime}}\left(0, T ; W^{-1, p^{\prime}}(\Omega)\right)} \leq C(k M)^{\frac{1}{p^{\prime}}} .
$$

Combining (2.10) and (2.11), we conclude that

$$
\|z\|_{W} \leq C \max \left\{(k M)^{\frac{1}{p}},(k M)^{\frac{1}{p^{\prime}}}\right\}
$$

where $M$ is defined in (2.7).

Step 3. Proof completed for nonnegative data.

Let us assume that $\mu \geq 0$ and $u_{0} \geq 0$; hence, we have $u_{t}-\Delta_{p} u \geq 0$, and $u \geq 0$ in $Q$. We claim that

$$
\left(T_{k}(u)\right)_{t}-\Delta_{p} T_{k}(u) \geq 0
$$

To prove (2.13), we consider the following smooth approximation of $T_{k}(u)$ : let us fix $\delta>0$ and define $S_{k, \delta}: \mathbb{R} \rightarrow \mathbb{R}$ by

$$
S_{k, \delta}(s)= \begin{cases}1 & \text { if }|s| \leq k \\ 0 & \text { if }|s|>k+\delta \\ \text { affine } & \text { otherwise }\end{cases}
$$

and finally, let us denote by $T_{k, \delta}: \mathbb{R} \rightarrow \mathbb{R}$ the primitive function of $S_{k, \delta}$, that is

$$
T_{k, \delta}(s)=\int_{0}^{s} S_{k, \delta}(\sigma) \mathrm{d} \sigma
$$

notice that $T_{k, \delta}(s)$ converges pointwise to $T_{k}(s)$ as $\delta$ goes to zero. 
Let $\varphi \in C_{c}^{\infty}(Q)$ be a nonnegative function and take $T_{k, \delta}^{\prime}(u) \varphi$ as test function in (2.2). We obtain, using that $\mu \geq 0$ and that $T_{k, \delta}(s)$ is concave for $s \geq 0$,

$$
-\int_{0}^{T} \varphi_{t} T_{k, \delta}(u) \mathrm{d} t+\int_{Q}|\nabla u|^{p-2} \nabla u \cdot \nabla \varphi S_{k, \delta}(u) \mathrm{d} x \mathrm{~d} t \geq 0,
$$

which yields (2.13) as $\delta$ goes to 0 .

Combining (2.8) and (2.13), we obtain

$$
-z_{t}-\Delta_{p} z \geq-\left(T_{k}(u)\right)_{t}-\Delta_{p} T_{k}(u)
$$

Since both $z$ and $T_{k}(u)$ belong to $L^{p}\left(0, T ; W_{0}^{1, p}(\Omega)\right)$, a standard comparison argument (multiply both sides of (2.16) by $\left(z-T_{k}(u)\right)^{-}$) allows us to conclude that $z \geq T_{k}(u)$ a.e. in $Q$. In particular, $z \geq k$ a.e. on $\{u>k\}$. On the other hand, since $u$ belongs to $W$, it has a unique cap-quasi continuous representative (still denoted by $u$ ); hence, the set $\{u>k\}$ is cap-quasi open, and its capacity can be estimated with Lemma 2.1. Therefore, we get

$$
\operatorname{cap}_{p}(\{u>k\}) \leq\left\|\frac{z}{k}\right\|_{W} .
$$

Using (2.12) we obtain (1.8).

Step 4. Comparison with $\mu^{+}$and $\mu^{-}$when $\mu$ is a smooth function.

Let us consider the case where $\mu \in C^{\infty}(\bar{Q})$. Then, $\mu^{+} \in \mathcal{M}(\Omega) \cap L^{p^{\prime}}(0, T$; $\left.W^{-1, p^{\prime}}(\Omega)\right)$ and we can consider the unique solution $v \in W$ of the problem

$$
\begin{cases}v_{t}-\Delta_{p} v=\mu^{+} & \text {in } Q, \\ v=u_{0}^{+} & \text {on }\{0\} \times \Omega, \\ v=0 & \text { on }(0, T) \times \partial \Omega .\end{cases}
$$

By comparison principle, we have $v \geq u$. Using Step 3, we deduce that there exists a nonnegative function $z \in W$ such that

$$
z \geq T_{k}(v) \geq T_{k}(u)
$$

and

$$
\|z\|_{W} \leq C \max \left\{k^{\frac{1}{p}}, k^{\frac{1}{p^{\prime}}}\right\}
$$

where $C=C\left(\|\mu\|_{\mathcal{M}(Q)},\left\|u_{0}\right\|_{L^{1}(\Omega)}, p\right)$. Similarly, using the solution of (1.7) with data $-\mu^{-}$and $-u_{0}^{-}$, we deduce that there exists a nonnegative function $w \in W$ such that

$$
T_{k}(u) \geq-w
$$

and

$$
\|w\|_{W} \leq C \max \left\{k^{\frac{1}{p}}, k^{\frac{1}{p^{\prime}}}\right\}
$$


We have thus proved that there exist two nonnegative functions $z, w \in W$ such that

$$
-w \leq T_{k}(u) \leq z \text { and }\|z\|_{W}+\|w\|_{W} \leq C \max \left\{k^{\frac{1}{p}}, k^{\frac{1}{p^{\prime}}}\right\},
$$

where $C$ depends on $\|\mu\|_{\mathcal{M}(Q)},\left\|u_{0}\right\|_{L^{1}(\Omega)}$ and $p$.

Step 5. Proof completed.

Let us fix $\theta \in C_{c}^{\infty}(Q)$ and set $\tilde{\mu}=\theta \mu$. By standard properties of convolutions (see e.g., [13, Lemma 2.25]), given a sequence of mollifiers $\left(\rho_{n}\right)$, we have $\rho_{n} * \tilde{\mu} \in C_{c}^{\infty}(Q)$,

$$
\rho_{n} * \tilde{\mu} \rightarrow \tilde{\mu} \quad \text { strongly in } L^{p^{\prime}}\left(0, T ; W^{-1, p^{\prime}}(\Omega)\right)
$$

and

$$
\left\|\rho_{n} * \tilde{\mu}\right\|_{\mathcal{M}(Q)} \leq\|\tilde{\mu}\|_{\mathcal{M}(Q)} \leq\|\mu\|_{\mathcal{M}(Q)} .
$$

Take now $\left(\theta_{j}\right)$ to be a sequence of $C_{c}^{\infty}(Q)$ functions such that $\theta_{j} \uparrow 1$ and consider the solutions $u_{j, n}$ of the problem

$$
\begin{cases}\left(u_{j, n}\right)_{t}-\Delta_{p} u_{j, n}=\rho_{n} *\left(\theta_{j} \mu\right) & \text { in } Q, \\ u_{j, n}=u_{0} & \text { on }\{0\} \times \Omega, \\ u_{j, n}=0 & \text { on }(0, T) \times \partial \Omega .\end{cases}
$$

As $n \rightarrow \infty$, the sequence $\left(u_{j, n}\right)$ converges in $L^{p}\left(0, T ; W_{0}^{1, p}(\Omega)\right)$ to the solution $u_{j}$ of (1.7) with $\theta_{j} \mu$ as datum. Next, as $j \rightarrow+\infty$,

$$
u_{j} \rightarrow u \text { in } L^{\infty}\left(0, T ; L^{1}(\Omega)\right)
$$

This is a consequence of a standard $L^{1}$-contraction argument. Indeed, subtracting equations (1.7) and (2.17), and taking $T_{1}\left(u_{j, n}-u\right)$ as test function, we get (note that both $u_{j, n}$ and $u$ belong to $W$ ),

$$
\begin{aligned}
& \int_{\Omega}\left|u_{j, n}-u\right|(t) \mathrm{d} x \\
& \quad \leq C\left\|\rho_{n} *\left(\theta_{j} \mu\right)-\theta_{j} \mu\right\|_{L^{p^{\prime}}\left(0, T ; W^{-1, p^{\prime}}(\Omega)\right)}\left\|T_{1}\left(u_{j, n}-u\right)\right\|_{L^{p}\left(0, T ; W_{0}^{1, p}(\Omega)\right)} \\
& \quad+C \int_{\Omega} T_{1}\left(u_{j, n}-u\right)\left(\theta_{j}-1\right) \mathrm{d} \mu,
\end{aligned}
$$

which yields

$$
\begin{aligned}
& \left\|\left(u_{j, n}-u\right)(t)\right\|_{L^{1}(\Omega)} \\
& \quad \leq C\left\|\rho_{n} *\left(\theta_{j} \mu\right)-\theta_{j} \mu\right\|_{L^{p^{\prime}}\left(0, T ; W^{-1, p^{\prime}}(\Omega)\right)}\left\|T_{1}\left(u_{j, n}-u\right)\right\|_{L^{p}\left(0, T ; W_{0}^{1, p}(\Omega)\right)} \\
& \quad+C\left\|\left(1-\theta_{j}\right) \mu\right\|_{\mathcal{M}(Q) .}
\end{aligned}
$$

Since for $j$ fixed $u_{j, n}$ is bounded in $L^{p}\left(0, T ; W_{0}^{1, p}(\Omega)\right)$, as $n \rightarrow+\infty$, the first term in the right-hand side tends to 0 , hence

$$
\left\|\left(u_{j}-u\right)(t)\right\|_{L^{1}(\Omega)} \leq C\left\|\left(1-\theta_{j}\right) \mu\right\|_{\mathcal{M}(Q)} .
$$


Since the latter term tends to zero as $j \rightarrow \infty$ by dominated convergence, we deduce the convergence of $u_{j}$ to $u$.

By Step 4, there exist nonnegative functions $z_{j, n}$ and $w_{j, n}$ such that

$$
-w_{j, n} \leq T_{k}\left(u_{j, n}\right) \leq z_{j, n}
$$

and

$$
\left\|z_{j, n}\right\|_{W}+\left\|w_{j, n}\right\|_{W} \leq C \max \left\{k^{\frac{1}{p}}, k^{\frac{1}{p^{\prime}}}\right\},
$$

where $C=C\left(\left\|\rho_{n} *\left(\theta_{j} \mu\right)\right\|_{\mathcal{M}(Q)},\left\|u_{0}\right\|_{L^{1}(\Omega)}, p\right)$. Since

$$
\left\|\rho_{n} *\left(\theta_{j} \mu\right)\right\|_{\mathcal{M}(Q)} \leq\|\mu\|_{\mathcal{M}(Q)},
$$

the constant $C$ can be chosen independently of $n$ and $j$. The sequences $\left(z_{j, n}\right)$ and $\left(w_{j, n}\right)$ being bounded in $W$, they converge weakly up to subsequences to nonnegative functions $z, w \in W$ and almost everywhere in $Q$. Thus,

$$
-w \leq T_{k}(u) \leq z \quad \text { a.e. in } Q
$$

and

$$
\|z\|_{W}+\|w\|_{W} \leq C \max \left\{k^{\frac{1}{p}}, k^{\frac{1}{p^{\prime}}}\right\},
$$

where $C=C\left(\|\mu\|_{\mathcal{M}(Q)},\left\|u_{0}\right\|_{L^{1}(\Omega)}, p\right)$. Since $u \in W$, it admits a uniquely defined cap-quasi continuous representative; hence, the sets $\{u>k\}$ and $\{u<-k\}$ are capquasi open. Using Lemma 2.1, we get

$$
\operatorname{cap}_{p}(\{|u|>k\}) \leq \operatorname{cap}_{p}(\{u>k\})+\operatorname{cap}_{p}(\{u<-k\}) \leq\left\|\frac{z}{k}\right\|_{W}+\left\|\frac{w}{k}\right\|_{W}
$$

which yields (1.8).

The same argument as above still holds for more general nonlinear operators. Consider, for example, the problem

$$
\begin{cases}u_{t}-\operatorname{div}(a(t, x, \nabla u))=\mu & \text { in } Q \\ u=u_{0} & \text { on }\{0\} \times \Omega, \\ u=0 & \text { on }(0, T) \times \partial \Omega,\end{cases}
$$

where $a: Q \times \mathbb{R}^{N} \rightarrow \mathbb{R}^{N}$ is a Carathéodory function (i.e., $a(\cdot, \cdot, \xi)$ is measurable on $Q$ for every $\xi$ in $\mathbb{R}^{N}$, and $a(t, x, \cdot)$ is continuous on $\mathbb{R}^{N}$ for almost every $(t, x)$ in $\left.Q\right)$, such that the following holds:

$$
\begin{aligned}
& a(t, x, \xi) \cdot \xi \geq \alpha|\xi|^{p}, \\
& |a(t, x, \xi)| \leq \beta\left[b(t, x)+|\xi|^{p-1}\right], \\
& {[a(t, x, \xi)-a(t, x, \eta)] \cdot(\xi-\eta)>0,}
\end{aligned}
$$

for almost every $(t, x)$ in $Q$, for every $\xi, \eta \in \mathbb{R}^{N}$, with $\xi \neq \eta$, where $p>1, \alpha$ and $\beta$ are two positive constants, and $b$ is a nonnegative function in $L^{p^{\prime}}(Q)$.

We obtain in a similar way the following capacitary estimate: 
THEOREM 2.2. Assume that (2.19)-(2.21) hold. Given $u_{0} \in L^{2}(\Omega)$ and $\mu \in$ $\mathcal{M}(Q) \cap L^{p^{\prime}}\left(0, T ; W^{-1, p^{\prime}}(\Omega)\right)$, let $u \in W$ be the (unique) weak solution of (2.18). Then,

$$
\operatorname{cap}_{p}(\{|u|>k\}) \leq C \max \left\{\frac{1}{k^{\frac{1}{p}}}, \frac{1}{k^{\frac{1}{p^{\prime}}}}\right\} \quad \forall k \geq 1,
$$

where $C>0$ is a constant depending on $\|\mu\|_{\mathcal{M}(Q)},\left\|u_{0}\right\|_{L^{1}(\Omega)},\|b\|_{L^{p^{\prime}}(Q)}, \alpha, \beta$ and $p$.

The proof runs exactly as before, replacing $\Delta_{p}$ with $\operatorname{div}(a(x, t, \nabla(\cdot)))$ and using in the standard way the coercivity condition (2.19) (e.g., in Step 1) and the growth condition (2.20) (e.g., to estimate the right-hand side in Step 2).

Let us stress that both Theorems 1.2 and 2.2 are meant to provide estimates for usual weak solutions, this is why we asked that $\mu \in L^{p^{\prime}}\left(0, T ; W^{-1, p^{\prime}}(\Omega)\right)$ and that $u_{0} \in L^{2}(\Omega)$ in the statements. However, the estimate only depends on the norm of $\mu$ as a measure and of $u_{0}$ in $L^{1}(\Omega)$. Indeed, in Section 4 , we will extend this result, in a suitable generalized form (see Proposition 4.8), by considering the larger framework of renormalized solutions.

\section{Properties of diffuse measures and the approximation result}

The representation result proved in [13] states the following: if $\mu$ is a diffuse measure, then there exist $f \in L^{1}(Q), g \in L^{p}(0, T ; V)$ and $\chi \in L^{p^{\prime}}\left(0, T ; W^{-1, p^{\prime}}(\Omega)\right)$ such that

$$
\mu=f+g_{t}+\chi \quad \text { in } \mathcal{D}^{\prime}(Q)
$$

The possibility that the above decomposition holds for some $g \in L^{\infty}(Q)$ has a special interest, as it was also pointed out in [19]. In particular, one has the following counterpart.

PROPOSITION 3.1. Assume that $\mu \in \mathcal{M}(Q)$ satisfies (3.1), where $f \in$ $L^{1}(Q), g \in L^{p}(0, T ; V)$ and $\chi \in L^{p^{\prime}}\left(0, T ; W^{-1, p^{\prime}}(\Omega)\right)$. If $g \in L^{\infty}(Q)$, then $\mu$ is diffuse.

Proof. Because of the inner regularity of $\mu$, it suffices to prove that for any compact set $K \subset Q$ such that $\operatorname{cap}_{p}(K)=0, \mu(K)=0$. Now, if $\operatorname{cap}_{p}(K)=0$, then by [13, Proposition 2.14] there exists a sequence of functions $\psi_{n} \in C_{c}^{\infty}(Q)$ such that $\psi_{n} \geq \chi_{K}$ and $\left\|\psi_{n}\right\|_{W} \rightarrow 0$.

Take a smooth function $\Phi: \mathbb{R} \rightarrow \mathbb{R}$ such that $\Phi(0)=0,0 \leq \Phi(s) \leq 1, \Phi(s)=1$ if $s \geq 1$ and $\Phi^{\prime}, \Phi^{\prime \prime}$ are bounded in $\mathbb{R}$. If we set $\xi_{n}=\Phi\left(\psi_{n}\right)$, then $\left(\xi_{n}\right)$ is a sequence of smooth functions such that $\xi_{n}=1$ on $K$ and $0 \leq \xi_{n} \leq 1$ in $Q$. Moreover, we have

$$
\xi_{n} \rightarrow 0 \text { in } L^{p}(0, T ; V) .
$$


Since $\left(\xi_{n}\right)_{t}=\Phi^{\prime}\left(\psi_{n}\right)\left(\psi_{n}\right)_{t}$, for every $\phi \in L^{\infty}(Q) \cap L^{p}(0, T ; V)$ with compact support in $Q$, we have

$$
\begin{aligned}
\left|\int_{Q}\left(\xi_{n}\right)_{t} \phi \mathrm{d} x \mathrm{~d} t\right| \leq & \left\|\left(\psi_{n}\right)_{t}\right\|_{L^{p^{\prime}}\left(0, T ; V^{\prime}\right)}\left\|\Phi^{\prime}\left(\psi_{n}\right) \phi\right\|_{L^{p}(0, T ; V)} \\
\leq & \left\|\left(\psi_{n}\right)_{t}\right\|_{L^{p^{\prime}}\left(0, T ; V^{\prime}\right)}\left(\|\phi\|_{L^{\infty}(Q)}\left\|\Phi^{\prime \prime}\right\|_{L^{\infty}(\mathbb{R})}\left\|\psi_{n}\right\|_{L^{p}(0, T ; V)}\right. \\
& \left.+\left\|\Phi^{\prime}\right\|_{L^{\infty}(\mathbb{R})}\|\phi\|_{L^{p}(0, T ; V)}\right) .
\end{aligned}
$$

By the strong convergence of $\left(\psi_{n}\right)$ in $L^{p}(0, T ; V)$ and of $\left(\left(\psi_{n}\right)_{t}\right)$ in $L^{p^{\prime}}\left(0, T ; V^{\prime}\right)$,

$$
\lim _{n \rightarrow \infty} \int_{Q}\left(\xi_{n}\right)_{t} \phi \mathrm{d} x \mathrm{~d} t=0 .
$$

Given $\varepsilon>0$, let $\omega \subset Q$ be an open set such that

$$
K \subset \omega \text { and }|\mu|(\omega \backslash K) \leq \varepsilon,
$$

and let $\varphi$ be a cut-off function for $K$ whose support is contained in $\omega$. We have

$$
\int_{K} \mathrm{~d} \mu=\int_{Q} \varphi \xi_{n} \mathrm{~d} \mu-\int_{\omega \backslash K} \varphi \xi_{n} \mathrm{~d} \mu
$$

so that

$$
|\mu(K)| \leq\left|\int_{Q} f \varphi \xi_{n} \mathrm{~d} x \mathrm{~d} t-\int_{Q} g\left(\varphi \xi_{n}\right)_{t} \mathrm{~d} x \mathrm{~d} t+\int_{0}^{T}\left\langle\chi, \varphi \xi_{n}\right\rangle\right| \mathrm{d} t+\varepsilon .
$$

It is easy to check, using (3.2), that both

$$
\int_{Q} f \varphi \xi_{n} \mathrm{~d} x \mathrm{~d} t \text { and } \int_{0}^{T}\left\langle\chi, \varphi \xi_{n}\right\rangle \mathrm{d} t
$$

go to zero as $n$ goes to infinity. Moreover, thanks to (3.3) and since $g \in L^{p}(0, T, V) \cap$ $L^{\infty}(Q)$, we deduce that

$$
\int_{Q} g\left(\varphi \xi_{n}\right)_{t}=\int_{Q} g(\varphi)_{t} \xi_{n}+\int_{Q} g \varphi\left(\xi_{n}\right)_{t} \rightarrow 0
$$

Therefore, as $n \rightarrow \infty$, we get $|\mu(K)| \leq \varepsilon$, and since $\varepsilon$ is arbitrary, this concludes the proof.

There exist diffuse measures whose time derivative part $g$ is essentially unbounded. The following example provides a typical case.

EXAMPLE 3.1. Given $0<t_{0}<T$, let

$$
\mu=\delta_{t_{0}} \otimes h
$$

where $h \in L^{1}(\Omega)$. By [13, Theorem 2.15], $\mu$ is diffuse. We claim that if (3.1) holds for some $g \in L^{\infty}(Q)$, then $h \in L^{\infty}(\Omega)$. 
For simplicity, let $p=2$ (the case $p \neq 2$ can be handled in a similar way by using the $p$-Laplacian) and let $u \in L^{2}\left(t_{0}, T ; H_{0}^{1}(\Omega)\right) \cap C\left(\left[t_{0}, T\right] ; L^{1}(\Omega)\right)$ be a solution of (see [22]),

$$
\begin{cases}u_{t}-\Delta u+|\nabla u|^{2}=0 & \text { in }\left(t_{0}, T\right) \times \Omega, \\ u=h & \text { on }\left\{t_{0}\right\} \times \Omega, \\ u=0 & \text { on }\left(t_{0}, T\right) \times \partial \Omega .\end{cases}
$$

Denoting by $\tilde{u}$ the extension of $u$ in $Q$ as identically zero on $\left(0, t_{0}\right) \times \Omega$, then $\tilde{u} \in L^{2}\left(0, T ; H_{0}^{1}(\Omega)\right)$ and

$$
\tilde{u}_{t}-\Delta \tilde{u}+|\nabla \tilde{u}|^{2}=\mu \text { in } Q .
$$

Let $f \in L^{1}(Q), g \in L^{p}(0, T ; V)$, and $\chi \in L^{p^{\prime}}\left(0, T ; W^{-1, p^{\prime}}(\Omega)\right)$ be such that

$$
\mu=f+g_{t}+\chi \quad \text { in } \mathcal{D}^{\prime}(Q)
$$

Since (3.4) also provides a decomposition of $\mu$, by [13, Lemma 2.29], we have

$$
\tilde{u}-g \in C\left([0, T] ; L^{1}(\Omega)\right) .
$$

Set $w=\tilde{u}-g$. Since $w \in C\left([0, T] ; L^{1}(\Omega)\right)$, we have for every $\varphi \in C_{c}^{\infty}(\Omega)$,

$$
\lim _{t \nearrow t_{0}} \int_{\Omega}|w(t, x) \varphi(x)| \mathrm{d} x=\int_{\Omega}\left|w\left(t_{0}, x\right) \varphi(x)\right| \mathrm{d} x .
$$

Since we have, for almost every $t \in\left(0, t_{0}\right)$,

$$
\int_{\Omega}|w(t, x) \varphi(x)| \mathrm{d} x=\int_{\Omega}|g(t, x) \varphi(x)| \mathrm{d} x \mathrm{~d} t \leq\|g\|_{L^{\infty}(Q)}\|\varphi\|_{L^{1}(\Omega)},
$$

it follows that

$$
\int_{\Omega}\left|w\left(t_{0}, x\right) \varphi(x)\right| \mathrm{d} x \leq\|g\|_{L^{\infty}(Q)}\|\varphi\|_{L^{1}(\Omega)} .
$$

Therefore, $w\left(t_{0}, \cdot\right) \in L^{\infty}(\Omega)$ and

$$
\left\|w\left(t_{0}, \cdot\right)\right\|_{L^{\infty}(\Omega)} \leq\|g\|_{L^{\infty}(Q)} .
$$

On the other hand, since $\tilde{u}=w+g$ on $Q$,

$$
\int_{\Omega}|\tilde{u}(t, x) \varphi(x)| \mathrm{d} x \leq \int_{\Omega}|w(t, x) \varphi(x)| \mathrm{d} x+\|g\|_{L^{\infty}(Q)}\|\varphi\|_{L^{1}(\Omega)},
$$

for all $\varphi \in C_{c}^{\infty}(\Omega)$. Hence, using the fact that $\tilde{u}=u \chi_{\left[t_{0}, T\right)}$ converges to $h$ as $t \searrow t_{0}$,

$$
\int_{\Omega}|h \varphi| \mathrm{d} x \leq \int_{\Omega}\left|w\left(t_{0}, x\right) \varphi(x)\right| \mathrm{d} x+\|g\|_{L^{\infty}(Q)}\|\varphi\|_{L^{1}(\Omega)} \leq 2\|g\|_{L^{\infty}(Q)}\|\varphi\|_{L^{1}(\Omega)},
$$

which implies that $h \in L^{\infty}(\Omega)$. 
In view of Example 3.1, Theorem 1.1 is actually the best one can expect, since it shows that the class of measures written as in (3.1) with $g$ bounded is dense (in the strong topology) in $\mathcal{M}_{0}(Q)$.

Before giving the proof of this result, we point out a few more things. First, it is often useful to work with measures compactly supported on the parabolic cylinder. The next lemma provides a tool for such situations. The proof of this result can be obtained as a straightforward modification of [13, Lemma 2.25].

LEMMA 3.1. Let $\mu \in \mathcal{M}_{0}(Q)$. Then, for every $\theta \in C_{c}^{\infty}(Q)$ such that $0 \leq \theta \leq 1$,

$$
\tilde{\mu}=\theta \mu
$$

is a diffuse measure with compact support on $Q$ such that

$$
\|\tilde{\mu}\|_{\mathcal{M}(Q)} \leq\|\mu\|_{\mathcal{M}(Q)} .
$$

Moreover, if $\mu=f+g_{t}+\chi$ is a decomposition of $\mu$ as in (3.1) with $\chi=\operatorname{div}(H)$, $H \in L^{p^{\prime}}(Q)$, then

$$
\tilde{\mu}=\tilde{f}+\tilde{g}_{t}+\tilde{\chi}
$$

with

$$
\begin{aligned}
& \tilde{f}=\theta f-H \cdot \nabla \theta-\theta_{t} g \in L^{1}(Q), \\
& \tilde{g}=\theta g \in L^{p}(0, T ; V), \\
& \tilde{\chi}=\operatorname{div}(\theta H) \in L^{p^{\prime}}\left(0, T ; W^{-1, p^{\prime}}(\Omega)\right) .
\end{aligned}
$$

In particular, if $g \in L^{\infty}(Q)$, then $\tilde{g} \in L^{\infty}(Q)$.

We will also need an important property enjoyed by the convolution of diffuse measures. We first recall the following definition (see [10] and also [18]):

DEFINITION 3.2. A sequence of measures $\left(\mu_{n}\right)$ in $Q$ is equidiffuse if for every $\varepsilon>0$ there exists $\eta>0$ such that for every Borel measurable set $E \subset Q$,

$$
\operatorname{cap}_{p}(E)<\eta \Longrightarrow\left|\mu_{n}\right|(E)<\varepsilon \quad \forall n \geq 1 \text {. }
$$

PROPOSITION 3.3. If $\mu \in \mathcal{M}_{0}(Q)$, then the sequence $\left(\rho_{n} * \mu\right)$ is equidiffuse.

Proof. It suffices to establish the result when $\mu \geq 0$; in the general case, we can apply the conclusion to the positive and the negative parts of $\mu$.

Assume by contradiction that $\left(\rho_{n} * \mu\right)$ is not equidiffuse. Passing to a subsequence if necessary, there exist $\varepsilon>0$ and a sequence $\left(E_{n}\right)$ of Borel subsets of $Q$ such that

$$
\operatorname{cap}_{p}\left(E_{n}\right) \leq \frac{1}{n} \text { and } \int_{E_{n}} \rho_{n} * \mu \geq \varepsilon
$$


for every $n \geq 1$. By definition of capacity, there exists an open subset $\omega_{n} \subset Q$ such that

$$
E_{n} \subset \omega_{n} \text { and } \operatorname{cap}_{p}\left(\omega_{n}\right) \leq \frac{2}{n} .
$$

Let $\left(\zeta_{n}\right)$ be a sequence in $W$ such that

$$
\zeta_{n} \geq \chi_{\omega_{n}} \text { a.e. and }\left\|\zeta_{n}\right\|_{W} \leq \frac{3}{n}
$$

Let $A \Subset Q$ be an open set such that $\mu(Q \backslash A)<\frac{\varepsilon}{2}$, and let $\varphi \in C_{c}^{\infty}(Q)$ be such that

$$
0 \leq \varphi \leq 1 \text { in } Q \text { and } \varphi=1 \text { on } A \text {. }
$$

Since $\varphi$ and $\zeta_{n}$ are nonnegative and $T_{1}$ is concave on $\mathbb{R}^{+}$,

$$
T_{1}\left(\zeta_{n}\right) \leq T_{1}\left(\varphi \zeta_{n}\right)+T_{1}\left((1-\varphi) \zeta_{n}\right)
$$

Given a sequence $\left(\rho_{n}\right)$ of mollifiers satisfying (2.1), for every $n \geq 1$, we have

$$
\begin{aligned}
\varepsilon \leq \int_{\omega_{n}} \rho_{n} * \mu \mathrm{d} x \mathrm{~d} t & \leq \int_{Q} T_{1}\left(\zeta_{n}\right)\left(\rho_{n} * \mu\right) \mathrm{d} x \mathrm{~d} t \\
& \leq \int_{Q} T_{1}\left(\varphi \zeta_{n}\right)\left(\rho_{n} * \mu\right) \mathrm{d} x \mathrm{~d} t+\int_{Q} T_{1}\left((1-\varphi) \zeta_{n}\right)\left(\rho_{n} * \mu\right) \mathrm{d} x \mathrm{~d} t \\
& \leq \int_{Q} \check{\rho}_{n} * T_{1}\left(\varphi \zeta_{n}\right) \mathrm{d} \mu+\int_{Q \backslash A} \rho_{n} * \mu \mathrm{d} x \mathrm{~d} t .
\end{aligned}
$$

Since $A$ is open, we have (see e.g., [15, Sect. 1.9, Theorem 1])

$$
\limsup _{n \rightarrow \infty} \int_{Q \backslash A} \rho_{n} * \mu \mathrm{d} x \mathrm{~d} t \leq \mu(Q \backslash A) .
$$

We now show that

$$
\lim _{n \rightarrow \infty} \int_{Q} \check{\rho}_{n} * T_{1}\left(\varphi \zeta_{n}\right) \mathrm{d} \mu \rightarrow 0 .
$$

Indeed, we have $\left\|\varphi \zeta_{n}\right\|_{W} \rightarrow 0$, and since $\varphi$ has compact support,

$$
\left\|\check{\rho}_{n} *\left(\varphi \zeta_{n}\right)\right\|_{W} \rightarrow 0
$$

Passing to a subsequence, there exists a Borel set $F \subset Q \operatorname{such}$ that $\operatorname{cap}_{p}(F)=0$ and

$$
\check{\rho}_{n} *\left(\varphi \zeta_{n}\right)(x) \rightarrow 0 \quad \forall x \in Q \backslash F
$$

Since the measure $\mu$ is diffuse, we deduce that $\left(\check{\rho}_{n} *\left(\varphi \zeta_{n}\right)\right)$ converges a.e. with respect to $\mu$. Since $\varphi \zeta_{n}$ is nonnegative,

$$
0 \leq \check{\rho}_{n} * T_{1}\left(\varphi \zeta_{n}\right) \leq \check{\rho}_{n} * \varphi \zeta_{n}
$$


Thus,

$$
\check{\rho}_{n} * T_{1}\left(\varphi \zeta_{n}\right) \rightarrow 0 \quad \mu \text {-a.e. }
$$

Assertion (3.6) is now a consequence of the dominated convergence theorem.

By (3.5)-(3.6), we deduce that

$$
\varepsilon \leq \mu(Q \backslash A)
$$

This contradicts our choice of $A$. Therefore, the sequence $\left(\rho_{n} * \mu\right)$ is equidiffuse.

We now present the

Proof of Theorem 1.1. Let $\mu_{n}=\rho_{n} * \mu$, where $\left(\rho_{n}\right)$ is a sequence of mollifiers satisfying (2.1). We denote by $u_{n}$ the solution of (1.7) with data $\mu_{n}$ and $u_{0}=0$. For $k>0$ and $\delta>0$, consider the functions $S_{k, \delta}$ and $T_{k, \delta}$ given by (2.14) and (2.15), respectively. Recall that $T_{k, \delta}$ converges pointwise to $T_{k}$ as $\delta \rightarrow 0$.

Given $\varphi \in C_{c}^{\infty}(Q)$, multiply the equation solved by $u_{n}$ by $S_{k, \delta}\left(u_{n}\right) \varphi$. We then have

$$
\begin{aligned}
& T_{k, \delta}\left(u_{n}\right)_{t}-\operatorname{div}\left(S_{k, \delta}\left(u_{n}\right)\left|\nabla u_{n}\right|^{p-2} \nabla u_{n}\right) \\
& \quad=S_{k, \delta}\left(u_{n}\right) \mu_{n}+\frac{1}{\delta}\left|\nabla u_{n}\right|^{p} \operatorname{sign}\left(u_{n}\right) \chi_{\left\{k \leq\left|u_{n}\right|<k+\delta\right\}} \operatorname{in} \mathcal{D}^{\prime}(Q) .
\end{aligned}
$$

Using $\left(1-S_{k, \delta}\left(u_{n}\right)\right) \operatorname{sign}\left(u_{n}\right)$ as test function in the Eq. (1.7) for $u_{n}$, we obtain

$$
\frac{1}{\delta} \int_{\left\{k \leq\left|u_{n}\right|<k+\delta\right\}}\left|\nabla u_{n}\right|^{p} \leq \int_{Q}\left|1-S_{k, \delta}\left(u_{n}\right)\right|\left|\mu_{n}\right| \leq \int_{\left\{\left|u_{n}\right|>k\right\}}\left|\mu_{n}\right| .
$$

Let us set

$$
v_{n}^{k}=\left(T_{k}\left(u_{n}\right)\right)_{t}-\Delta_{p}\left(T_{k}\left(u_{n}\right)\right)
$$

Thanks to (3.8), the right-hand side of (3.7) remains bounded in $L^{1}(Q)$ as $\delta \rightarrow 0$, then we deduce that $v_{n}^{k}$ is a finite measure in $Q$ and

$$
\begin{aligned}
\int_{Q}\left|v_{n}^{k}\right| & \leq \liminf _{\delta \rightarrow 0}\left\{\int_{Q}\left|S_{k, \delta}\left(u_{n}\right) \mu_{n}\right|+\frac{1}{\delta} \int_{\left\{k \leq\left|u_{n}\right|<k+\delta\right\}}\left|\nabla u_{n}\right|^{p}\right\} \\
& \leq \int_{\left\{\left|u_{n}\right| \leq k\right\}}\left|\mu_{n}\right|+\int_{\left\{\left|u_{n}\right|>k\right\}}\left|\mu_{n}\right|=\left\|\mu_{n}\right\|_{\mathcal{M}(Q)} \leq\|\mu\|_{\mathcal{M}(Q)} .
\end{aligned}
$$

In particular, $\left(v_{n}^{k}\right)$ remains uniformly bounded in $\mathcal{M}(Q)$ as $n \rightarrow \infty$.

We now show that

$$
v_{n}^{k} \stackrel{*}{\rightarrow} v^{k} \quad \text { weakly* in } \mathcal{M}(Q),
$$

where

$$
v^{k}=\left(T_{k}(u)\right)_{t}-\Delta_{p}\left(T_{k}(u)\right) .
$$


To this purpose, we recall that by classical results on parabolic equations with measure data (see Proposition 4.6 below), there exists a function $u \in L^{1}(Q)$ such that (taking a subsequence if necessary) $u_{n} \rightarrow u$ and $\nabla u_{n} \rightarrow \nabla u$ a.e. on $Q$. In particular, since $\left|\nabla T_{k}\left(u_{n}\right)\right|^{p-2} \nabla T_{k}\left(u_{n}\right)$ is bounded in $L^{p^{\prime}}(Q)$ and almost everywhere converges to $\left|\nabla T_{k}(u)\right|^{p-2} \nabla T_{k}(u)$, then it weakly converges to the same limit in $L^{p^{\prime}}(Q)$. Therefore, we have

$$
T_{k}\left(u_{n}\right) \rightarrow T_{k}(u) \quad \text { weakly in } L^{p}\left(0, T ; W_{0}^{1, p}(\Omega)\right)
$$

and

$$
\Delta_{p} T_{k}\left(u_{n}\right) \rightarrow \Delta_{p} T_{k}(u) \quad \text { weakly in } L^{p^{\prime}}\left(0, T ; W^{-1, p^{\prime}}(\Omega)\right)
$$

as $n \rightarrow \infty$. Together with the fact that $\left(v_{n}^{k}\right)$ is uniformly bounded in $\mathcal{M}(Q)$, this implies (3.9). Moreover, since $T_{k}(u) \in L^{p}\left(0, T ; W_{0}^{1, p}(\Omega)\right) \cap L^{\infty}(Q)$, it follows from Proposition 3.1 that $\nu^{k}$ is a diffuse measure.

By (3.7)-(3.8), we also have

$$
\begin{aligned}
\int_{Q}\left|v_{n}^{k}-\mu_{n}\right| & \leq \liminf _{\delta \rightarrow 0} \int_{Q}\left|S_{k, \delta}\left(u_{n}\right) \mu_{n}+\frac{\left|\nabla u_{n}\right|^{p}}{\delta} \operatorname{sign}\left(u_{n}\right) \chi_{\left\{k \leq\left|u_{n}\right|<k+\delta\right\}}-\mu_{n}\right| \\
& \leq \int_{\left\{\left|u_{n}\right|>k\right\}}\left|\mu_{n}\right|+\limsup _{\delta \rightarrow 0} \int_{\left\{k \leq\left|u_{n}\right|<k+\delta\right\}} \frac{\left|\nabla u_{n}\right|^{p}}{\delta} \leq 2 \int_{\left\{\left|u_{n}\right|>k\right\}}\left|\mu_{n}\right| .
\end{aligned}
$$

Recall that by Proposition 3.3, the sequence $\left(\mu_{n}\right)$ is equidiffuse. Applying Theorem 1.2, we can fix $k>0$ sufficiently large (depending only on $\varepsilon>0$ ) so that the right-hand side of (3.10) is $\leq \varepsilon, \forall n \geq 1$. From (3.10) and the lower semicontinuity of the norm with respect to the weak* convergence, we obtain

$$
\left\|v^{k}-\mu\right\| \mathcal{M}(Q) \leq \liminf _{n \rightarrow \infty} \int_{Q}\left|v_{n}^{k}-\mu_{n}\right| \leq 2 \liminf _{n \rightarrow \infty} \int_{\left\{\left|u_{n}\right|>k\right\}}\left|\mu_{n}\right| \leq \varepsilon .
$$

This concludes the proof of Theorem 1.1.

\section{Renormalized formulation}

In this section, we come back to the construction of the approximation in the proof of Theorem 1.1, and we develop that idea in connection with a renormalized formulation for solutions of (1.11) when $\mu$ is a diffuse measure. Because of the intrinsic interest of renormalized formulations with measure data, we deal with the more general initial boundary value problem

$$
\begin{cases}u_{t}-\operatorname{div}(a(t, x, \nabla u))=\mu & \text { in } Q \\ u=u_{0} & \text { on }\{0\} \times \Omega \\ u=0 & \text { on }(0, T) \times \partial \Omega .\end{cases}
$$


In all the following, we assume that $a: Q \times \mathbb{R}^{N} \rightarrow \mathbb{R}^{N}$ satisfies (2.19)-(2.21), that

$$
u_{0} \in L^{1}(\Omega)
$$

and that $\mu$ is a diffuse measure, i.e.,

$$
\mu \in \mathcal{M}_{0}(Q) .
$$

A notion of renormalized solution for problem (4.1) when $\mu$ is a diffuse measure was introduced in [13], and in the same paper, the existence and uniqueness of such a solution are proved. In [14], a similar notion of entropy solution is also defined and proved to be equivalent to that of renormalized solution. Our goal is to give here a new definition which, in contrast with the previous ones, is not formulated in terms of a decomposition of $\mu$ like in (1.5). The next definition is certainly closer to the one used for conservation laws in [3] and to one of the existing formulations in the elliptic case (see $[11,12])$.

DEFINITION 4.1. Let $\mu \in \mathcal{M}_{0}(Q)$. A function $u \in L^{1}(Q)$ is a renormalized solution of problem (4.1) if $T_{k}(u) \in L^{p}\left(0, T ; W_{0}^{1, p}(\Omega)\right)$ for every $k>0$ and if there exists a sequence $\left(\lambda_{k}\right)$ in $\mathcal{M}(Q)$ such that

$$
\lim _{k \rightarrow \infty}\left\|\lambda_{k}\right\|_{\mathcal{M}(Q)}=0
$$

and

$$
\begin{array}{r}
-\int_{Q} T_{k}(u) \varphi_{t} \mathrm{~d} x \mathrm{~d} t+\int_{Q} a\left(t, x, \nabla T_{k}(u)\right) \nabla \varphi \mathrm{d} x \mathrm{~d} t \\
=\int_{Q} \varphi \mathrm{d} \mu+\int_{Q} \varphi \mathrm{d} \lambda_{k}+\int_{\Omega} T_{k}\left(u_{0}\right) \varphi(0, x) \mathrm{d} x
\end{array}
$$

for every $k>0$ and $\varphi \in C_{c}^{\infty}([0, T) \times \Omega)$.

REMARK 4.1. It is straightforward to check that, by approximation, one can take as test function in (4.3) any $\varphi \in W^{1, \infty}(Q)$ such that $\varphi=0$ on $(\{T\} \times \Omega) \cup((0, T) \times \partial \Omega)$.

Some considerations are in order concerning Definition 4.1. First of all, observe that (4.3) implies that $\left(T_{k}(u)\right)_{t}-\operatorname{div}\left(a\left(t, x, \nabla T_{k}(u)\right)\right)$ is a finite measure and

$$
\left(T_{k}(u)\right)_{t}-\operatorname{div}\left(a\left(t, x, \nabla T_{k}(u)\right)\right)=\mu+\lambda_{k} \quad \text { in } \mathcal{M}(Q) .
$$

This provides a decomposition of the measure $\mu+\lambda_{k}$ of the form (3.1) with $g \in L^{\infty}(Q)$. In view of Proposition 3.1, the left hand side is a diffuse measure. Since $\mu$ itself is diffuse, the consequence is that the measures $\lambda_{k}$ are diffuse. Moreover, condition (4.2) implies that the right-hand side is a strong approximation of $\mu$. In particular, the existence of a renormalized solution in the sense of Definition 4.1 implies as a corollary the statement of Theorem 1.1. 
Finally, since $T_{k}(u) \in L^{p}(0, T ; V)$, we have $\left(T_{k}(u)\right)_{t} \in W^{\prime 1}$ and, due to assumption (2.20), $a\left(t, x, \nabla T_{k}(u)\right) \in L^{p^{\prime}}(Q)$. Therefore, we conclude that

$$
\left(T_{k}(u)\right)_{t}-\operatorname{div}\left(a\left(t, x, \nabla T_{k}(u)\right)\right) \in W^{\prime} \cap \mathcal{M}(Q) .
$$

Another important fact is that we can recover from Eq. (4.3) the standard estimates known for nonlinear potentials. In order to prove such estimates and further properties of the renormalized formulation, we will need a few technical ingredients.

First of all, recall that any $z \in W$ admits a unique cap-quasi continuous representative; henceforth, by identifying $z$ with its cap-quasi continuous representative, the integral $\int_{Q} z d \mu$ is well defined for every diffuse measure $\mu$ and every $z \in W$. Unfortunately, given $z \in W$, a smooth truncation of $z$ need not belong to $W$. In this case, one is led to consider the larger space

$$
S=\left\{z \in L^{p}(0, T ; V) ; z_{t} \in L^{p^{\prime}}\left(0, T ; W^{-1, p^{\prime}}(\Omega)\right)+L^{1}(Q)\right\}
$$

endowed with its norm

$$
\|z\|_{S}=\|z\|_{L^{p}(0, T ; V)}+\left\|z_{t}\right\|_{L^{p^{\prime}}\left(0, T ; W^{-1, p^{\prime}}(\Omega)\right)+L^{1}(Q)} .
$$

Indeed,

LEMMA 4.1. For every $v \in W$ and every function $\Phi \in C^{2}(\mathbb{R})$ such that $\Phi^{\prime}$ and $\Phi^{\prime \prime}$ are bounded, we have $\Phi(v) \in S$ and the application $v \mapsto \Phi(v)$ is continuous from $W$ to $S$. Moreover, if $v_{t}=\operatorname{div}(G)+g$ with $G \in L^{p^{\prime}}(Q), g \in L^{p^{\prime}}\left(0, T ; L^{2}(\Omega)\right)$, then we have

$$
(\Phi(v))_{t}=\operatorname{div}\left(\Phi^{\prime}(v) G\right)+\Phi^{\prime}(v) g-\Phi^{\prime \prime}(v) G \cdot \nabla v \text { in } \mathcal{D}^{\prime}(Q) .
$$

Proof. It follows from [13, Theorem 2.11] that $C_{c}^{\infty}([0, T] \times \Omega)$ is dense in $W$. Then, there exists a sequence of smooth functions $\left(v_{n}\right)$ converging to $v$ in $W$. Let $v_{t}=$ $\operatorname{div}(G)+g$ with $G \in L^{p^{\prime}}(Q), g \in L^{p^{\prime}}\left(0, T ; L^{2}(\Omega)\right)$; the convergence of $\left(v_{n}\right)$ to $v$ in $W$ implies that $v_{n} \rightarrow v$ in $L^{p}(0, T ; V)$, and there exist $G_{n} \in L^{p^{\prime}}(Q)$ and $g_{n} \in L^{p^{\prime}}\left(0, T ; L^{2}(\Omega)\right)$ such that $\left(v_{n}\right)_{t}=\operatorname{div}\left(G_{n}\right)+g_{n}$ and $G_{n} \rightarrow G$ in $L^{p^{\prime}}(Q)$ and $g_{n} \rightarrow g$ in $L^{p^{\prime}}\left(0, T ; L^{2}(\Omega)\right)$. Since the equality (4.4) is true for $v_{n}$, passing to the limit (which is possible thanks to the properties of $\Phi$ ), we recover (4.4) for $v$. The continuity of $T$ is established in a similar way using identity (4.4).

${ }^{1}$ Indeed, for every $v \in L^{p}(0, T ; V)$, we have $v_{t} \in W^{\prime}$ in the following sense:

$$
\left\langle v_{t}, \varphi\right\rangle_{\left(W^{\prime}, W\right)}=-\int_{0}^{T}\left\langle v, \varphi_{t}\right\rangle_{\left(V, V^{\prime}\right)} \mathrm{d} t \quad \text { for every } \varphi \in W
$$

and since

$$
\left|\int_{0}^{T}\left\langle v, \varphi_{t}\right\rangle_{\left(V, V^{\prime}\right)} \mathrm{d} t\right| \leq\|v\|_{L^{p}(0, T ; V)}\left\|\varphi_{t}\right\|_{L^{p^{\prime}\left(0, T ; V^{\prime}\right)}} \leq\|v\|_{L^{p}(0, T ; V)}\|\varphi\|_{W}
$$

we have $v_{t} \in W^{\prime}$ and $\left\|v_{t}\right\|_{W^{\prime}} \leq\|v\|_{L^{p}(0, T ; V)}$. 
Bounded functions in $S$ satisfy a capacitary estimate for the parabolic capacity; we refer the reader to [19, Theorem 3 and Lemma 2] for a proof of the following

LEMMA 4.2. If $z \in S \cap L^{\infty}(Q)$, then $z$ admits a unique cap-quasi continuous representative. Moreover, we have

$$
\operatorname{cap}_{p}(\{|z|>k\}) \leq \frac{C}{k} \max \left\{[z]^{\frac{1}{p}},[z]^{\frac{1}{p^{\prime}}}\right\},
$$

where

$$
\begin{aligned}
& {[z]=\inf \left\{\|z\|_{L^{p}\left(0, T ; W_{0}^{1, p}(\Omega)\right)}^{p}+\left\|\left(z_{t}\right)_{1}\right\|_{L^{p^{\prime}}\left(0, T ; W^{-1, p^{\prime}}(\Omega)\right)}^{p^{\prime}}\right.} \\
& \left.+\|z\|_{\infty}\left\|\left(z_{t}\right)_{2}\right\|_{L^{1}(Q)}+\|z\|_{L^{\infty}\left(0, T ; L^{2}(\Omega)\right)}^{2}\right\}
\end{aligned}
$$

and the infimum is taken over all decompositions of $z_{t}=\left(z_{t}\right)_{1}+\left(z_{t}\right)_{2}$ with $\left(z_{t}\right)_{1} \in$ $L^{p^{\prime}}\left(0, T ; W^{-1, p^{\prime}}(\Omega)\right)$ and $\left(z_{t}\right)_{2} \in L^{1}(Q)$.

We obtain in particular the following corollary that allows one to pass from an inequality almost everywhere to an inequality cap-quasi everywhere.

COROLLARY 4.3. If $v \in W$ and $v \leq M$ almost everywhere in $Q$, then $v \leq M$ cap-quasi everywhere in $Q$.

Proof. Let us take a bounded, nondecreasing function $\Phi \in C^{2}(\mathbb{R})$ such that $\Phi^{\prime}, \Phi^{\prime \prime}$ are bounded, $\Phi(s) \equiv 0$ if $s \leq 0$ and $\Phi(s)>0$ if $s>0$. By Lemma 4.1, we deduce that $\Phi(v-M) \in S \cap L^{\infty}(Q)$. Since $v-M \leq 0$ almost everywhere, then $\Phi(v-M)=0$ almost everywhere. It follows from Lemma 4.2 that the unique cap-quasi continuous representative of $\Phi(v-M)$ is the function identically zero, that is $\Phi(v-M)=0$ cap-quasi everywhere. Therefore $v \leq M$ cap-quasi everywhere.

We now study the pointwise convergence of sequences in $S$ :

LEMMA 4.4. For every bounded sequence $\left(z_{n}\right)$ in $S \cap L^{\infty}(Q)$, if $z_{n} \rightarrow z$ in $S$, then there exists a subsequence $\left(z_{n_{k}}\right)$ converging to $z$ cap-quasi everywhere.

Proof. This is a classical argument; we present it here for the convenience of the reader. Since $\left(z_{n}\right)$ is bounded in $L^{\infty}(Q)$ and $z_{n} \rightarrow z$ in $S$, we have (with the notations of Lemma 4.2)

$$
\left[z_{n}-z\right] \rightarrow 0
$$

Take a subsequence $\left(z_{n_{k}}\right)$ such that

$$
\sum_{k=1}^{\infty} 2^{k} \max \left\{\left[z_{n_{k}}-z\right]^{\frac{1}{p}},\left[z_{n_{k}}-z\right]^{\frac{1}{p^{\prime}}}\right\}<\infty .
$$


Define the sets

$$
E_{k}=\left\{(x, t) \in Q:\left|z_{n_{k}}-z\right|>2^{-k}\right\}, \quad F_{m}=\bigcup_{k=m}^{\infty} E_{k}, \quad F_{\infty}=\bigcap_{m=0}^{\infty} F_{m} .
$$

By the subadditivity of the parabolic capacity and by the capacitary estimate from Lemma 4.2, we have

$$
\operatorname{cap}_{p}\left(F_{m}\right) \leq \sum_{k=m}^{\infty} \operatorname{cap}_{p}\left(E_{k}\right) \leq C \sum_{k=m}^{\infty} 2^{k} \max \left\{\left[z_{n_{k}}-z\right]^{\frac{1}{p}},\left[z_{n_{k}}-z\right]^{\frac{1}{p^{\prime}}}\right\} .
$$

Hence, $\operatorname{cap}_{p}\left(F_{m}\right) \rightarrow 0$, which implies cap $\operatorname{cop}_{\infty}\left(F_{\infty}\right)=0$. Since $z_{n_{k}}(x) \rightarrow z(x)$ for every $x \in Q \backslash F_{\infty}$, the conclusion follows.

We can now show how to extend the class of test functions in (4.3).

PROPOSITION 4.2. If $u$ is a renormalized solution of (4.1), then

$$
\begin{gathered}
-\int_{0}^{T}\left\langle T_{k}(u), v_{t}\right\rangle \mathrm{d} t+\int_{Q} a\left(t, x, \nabla T_{k}(u)\right) \nabla v \mathrm{~d} x \mathrm{~d} t \\
=\int_{Q} v \mathrm{~d} \mu+\int_{Q} v \mathrm{~d} \lambda_{k}+\int_{\Omega} T_{k}\left(u_{0}\right) v(0, x) \mathrm{d} x
\end{gathered}
$$

for every $v \in W \cap L^{\infty}(Q)$ such that $v=0$ on $\{T\} \times \Omega$.

Proof. Since $C_{c}^{\infty}([0, T] \times \Omega)$ is dense in $W$, there exists a sequence $\left(v_{n}\right)$ in $C_{c}^{\infty}([0, T] \times \Omega)$ converging to $v$ in $W$. Let $M$ such that $\|v\|_{L^{\infty}(Q)} \leq M$, and take a function $\Phi: \mathbb{R} \rightarrow \mathbb{R}$ which is $C^{\infty}$ and such that $\Phi(s)=s$ when $|s|<2 M$ and $\Phi^{\prime}$ has compact support. By Lemma 4.1, we have that $\Phi\left(v_{n}\right) \rightarrow \Phi(v)=v$ in $S$. Let us then call $w_{n}=\Phi\left(v_{n}\right)$. Note that $w_{n}$ is not an admissible function since it need not vanish on $\{T\} \times \Omega$. For this purpose, choose $\varphi=w_{n} \xi$ in (4.3) where $\xi \in W^{1, \infty}(0, T)$ and is compactly supported in $[0, T) ; \varphi$ can be used as a test function in view of Remark 4.1. We get

$$
\begin{aligned}
& -\int_{Q} T_{k}(u) w_{n} \xi_{t} \mathrm{~d} x \mathrm{~d} t-\int_{Q} T_{k}(u) \xi\left(w_{n}\right)_{t} \mathrm{~d} x \mathrm{~d} t+\int_{Q} a\left(t, x, \nabla T_{k}(u)\right) \nabla w_{n} \xi \mathrm{d} x \mathrm{~d} t \\
& =\int_{Q} w_{n} \xi \mathrm{d} \mu+\int_{Q} w_{n} \xi \mathrm{d} \lambda_{k}+\int_{\Omega} T_{k}\left(u_{0}\right) w_{n}(0, x) \xi(0) \mathrm{d} x .
\end{aligned}
$$

Since $w_{n} \rightarrow v$ in $S$ and $\left(w_{n}\right)$ is uniformly bounded, we can pass to the limit in the left hand side. Moreover, since $v_{n} \rightarrow v$ in $W$, there exists a subsequence such that $v_{n} \rightarrow v$ cap-quasi everywhere, hence $\mu$-a.e. and $\lambda_{k}$-a.e., since the two measures are diffuse. Being $\Phi$ smooth, we have $w_{n} \rightarrow \Phi(v)=v \mu$-a.e. and $\lambda_{k}$-a.e. Since $\left(w_{n}\right)$ is uniformly bounded, we can pass to the limit in the right-hand side by dominated convergence, obtaining

$$
\begin{aligned}
& -\int_{Q} T_{k}(u) v \xi_{t} \mathrm{~d} x \mathrm{~d} t-\int_{0}^{T}\left\langle T_{k}(u) \xi, v_{t}\right\rangle d t+\int_{Q} a\left(t, x, \nabla T_{k}(u)\right) \nabla v \xi \mathrm{d} x \mathrm{~d} t \\
& \quad=\int_{Q} v \xi \mathrm{d} \mu+\int_{Q} v \xi \mathrm{d} \lambda_{k}+\int_{\Omega} T_{k}\left(u_{0}\right) v(0, x) \xi(0) \mathrm{d} x .
\end{aligned}
$$


Given $\varepsilon \in(0, T)$, we now apply this identity with the function $\xi_{\varepsilon}:[0, T] \rightarrow \mathbb{R}$ given by $\xi_{\varepsilon}(t)=1-\frac{(t-T+\varepsilon)^{+}}{\varepsilon}$; in particular, $\xi_{\varepsilon}=1$ in $(0, T-\varepsilon), \xi_{\varepsilon}(T)=0$, and $\xi_{\varepsilon} \rightarrow 1$. Since $v \in C^{0}\left([0, T] ; L^{2}(\Omega)\right)$ and $v(T)=0$, we have

$\left|\int_{Q} T_{k}(u) v\left(\xi_{\varepsilon}\right)_{t} \mathrm{~d} x \mathrm{~d} t\right| \leq \frac{1}{\varepsilon} \int_{T-\varepsilon}^{T} \int_{\Omega}\left|T_{k}(u) v\right| \mathrm{d} x \mathrm{~d} t \leq k \frac{1}{\varepsilon} \int_{T-\varepsilon}^{T}\|v(t)\|_{L^{1}(\Omega)} \mathrm{d} t \rightarrow 0$.

By dominated convergence we can pass to the limit in all other terms; hence, we deduce (4.5).

One of the main roles of Proposition 4.2 is to give us a way to use test functions of the form $\psi(u)$. A difficulty arises from the fact that $u$ (and also $T_{k}(u)$ ) may not have a cap-quasi continuous representative. In order to overcome this point, our strategy will be to use approximations through so-called Steklov time-averages for functions $z:(0, T) \times \Omega \rightarrow \mathbb{R}$. More precisely, given $\varepsilon \in(0, T)$, define for every $h \in(0, \varepsilon)$ the functions $z_{h}:(0, T-\varepsilon) \times \Omega \rightarrow \mathbb{R}$ and $z_{-h}:[\varepsilon, T] \times \Omega \rightarrow \mathbb{R}$ by

$$
z_{h}(t, x)=\frac{1}{h} \int_{t}^{t+h} z(s, x) \mathrm{d} s \text { and } z_{-h}(t, x)=\frac{1}{h} \int_{t-h}^{t} z(s, x) \mathrm{d} s .
$$

In the context of parabolic equations, we can deal with Steklov averages as follows. Given $w: Q \rightarrow \mathbb{R}$, if $\varphi$ is a function with compact support in $[0, T) \times \Omega$, then $w_{h} \varphi$ has a meaning in $Q$, even though the function $w_{h}$ is not defined on $[T-h, T] \times \Omega$. Concerning $w_{-h} \varphi$, one can consider this function on the parabolic cylinder $(\epsilon, T) \times \Omega$ for every $h \in(0, \epsilon)$. Once we get the desired estimates independently of the parameter $h$, we can let $h \rightarrow 0$ and then $\epsilon \rightarrow 0$. Another approach consists in taking a sequence of smooth functions $\left(w_{0, j}\right)$ converging in $L^{1}(\Omega)$ to $w(0, \cdot)$, and for each $j$, we take an extension of $w(t, \cdot)$ as $w_{0, j}$ if $t<0$; once we get uniform estimates with respect to $j$, we can let $j \rightarrow \infty$.

Observe that, for every $z \in L^{p}(0, T ; V)$, we have $z_{h} \in W$; hence, $z_{h}$ admits a cap-quasi continuous representative. In addition, whenever $z \in L^{\infty}(Q)$, we have $\left|z_{h}\right| \leq\|z\|_{\infty}$ q.e. (i.e., except of a set of zero capacity). Indeed, for any $M$ such that $|z| \leq M$ a.e., we also have $\left|z_{h}\right| \leq M$ a.e., and since $z_{h} \in W$, we deduce from Corollary 4.3 that $\left|z_{h}\right| \leq M$ q.e. as well. Some further property of the Steklov averages with respect to capacity will be useful. For instance,

LEMMA 4.5. Let $z \in S$. Then, for every $\psi \in C_{c}^{\infty}(0, T)$,

$$
z_{h} \psi \rightarrow z \psi \quad \text { in } S
$$

If in addition $z \in L^{\infty}(Q)$, then for every sequence $\left(h_{n}\right)$ of positive numbers converging to 0 , there exists a subsequence $\left(h_{n_{k}}\right)$ such that

$$
z_{h_{n_{k}}} \rightarrow z \text { q.e. in } Q \text {. }
$$


Proof. If $\psi \in C_{c}^{\infty}(0, T)$, then $z_{h} \psi \in S$. In particular, if $z_{t}=\operatorname{div}(G)+g$, with $G \in L^{p^{\prime}}(Q), g \in L^{1}(Q)$, since $\psi$ has compact support in $(0, T)$, we have

$$
\left(z_{h} \psi\right)_{t}=\operatorname{div}\left(G_{h} \psi\right)+g_{h} \psi+z_{h} \psi_{t}
$$

where $G_{h}$ and $g_{h}$ are Steklov averages of $G$ and $g$, respectively. Since $G_{h} \rightarrow G$ in $L^{p^{\prime}}(Q), g_{h} \rightarrow g$ in $L^{1}(Q)$, and $z_{h} \rightarrow z$ in $L^{1}(Q)$, we have that $\left(z_{h} \psi\right)_{t}$ converges to $(z \psi)_{t}$ in $L^{p^{\prime}}\left(0, T ; W^{-1, p^{\prime}}(\Omega)\right)+L^{1}(Q)$; on the other hand, $z_{h}$ converges to $z$ in $L^{p}(0, T ; V)$ so that $z_{h} \psi \rightarrow z \psi$ in $L^{p}(0, T ; V)$. Therefore, we conclude that $z_{h} \psi \rightarrow z \psi$ in $S$. If in addition $z \in L^{\infty}(Q)$, then $\left(z_{h_{n}} \psi\right)$ is bounded in $S \cap L^{\infty}(Q)$ and, from Lemma 4.4, we conclude that $z_{h_{n}} \psi$ admits a subsequence converging cap-quasi everywhere. We now take $\psi_{j} \in C_{c}^{\infty}(0, T)$ such that $\psi_{j}=1$ in $\left(\frac{1}{j}, T-\frac{1}{j}\right)$; there exists a subsequence $\left(h_{n_{j}}\right)$ and a set $F_{j} \subset Q$ such that $\operatorname{cap}_{p}\left(F_{j}\right)=0$ and $z_{h_{n_{j}}} \rightarrow z$ in $\left(\left(\frac{1}{j}, T-\frac{1}{j}\right) \times \Omega\right) \backslash F_{j}$. Using a diagonal argument, we can construct a subsequence $\left(h_{n_{k}}\right)$ such that $z_{h_{n_{k}}} \rightarrow z$ in $Q \backslash F$, where $F=\bigcup_{j=1}^{\infty} F_{j}$ and $\operatorname{cap}_{p}(F)=0$.

The idea of using Steklov averages in connection with the renormalized formulation is developed in [5]. Following this latter paper, we deduce in particular the following result:

LEMMA 4.6. If $u$ is a renormalized solution of (4.1), then

$$
\begin{aligned}
& -\int_{Q} \Psi\left(T_{k}(u)\right) \xi_{t} \mathrm{~d} x \mathrm{~d} t-\int_{\Omega} \Psi\left(T_{k}\left(u_{0}\right)\right) \xi(0, x) \mathrm{d} x \\
& \quad+\int_{Q} a\left(t, x, \nabla T_{k}(u)\right) \nabla\left(\psi\left(T_{k}(u)\right) \xi\right) \mathrm{d} x \mathrm{~d} t \\
& \quad \leq \int_{Q}\left(\psi\left(T_{k}(u)\right)\right)_{h} \xi \mathrm{d} \mu+\|\psi\|_{\infty}\|\xi\|_{\infty}\left\|\lambda_{k}\right\|+o(1)_{h},
\end{aligned}
$$

and

$$
\begin{aligned}
& -\int_{Q} \Psi\left(T_{k}(u)\right) \xi_{t} \mathrm{~d} x \mathrm{~d} t-\int_{\Omega} \Psi\left(T_{k}\left(u_{0}\right)\right) \xi(0, x) \mathrm{d} x \\
& \quad+\int_{Q} a\left(t, x, \nabla T_{k}(u)\right) \nabla\left(\psi\left(T_{k}(u)\right) \xi\right) \mathrm{d} x \mathrm{~d} t \\
& \quad \geq \int_{Q}\left(\psi\left(T_{k}(u)\right)\right)_{-h} \xi \mathrm{d} \mu-\|\psi\|_{\infty}\|\xi\|_{\infty}\left\|\lambda_{k}\right\|-o(1)_{h},
\end{aligned}
$$

for every nondecreasing $\psi \in W^{1, \infty}(\mathbb{R})$ and every nonnegative $\xi \in C_{c}^{\infty}([0, T) \times \bar{\Omega})$ such that $\psi(0) \xi=0$ on $(0, T) \times \partial \Omega$, where $\Psi(r)=\int_{0}^{r} \psi(s) \mathrm{d} s$ and $o(1)_{h} \rightarrow 0$ as $h \rightarrow 0$.

Proof. We choose in (4.5)

$$
v(t, x)=\xi\left(\psi\left(T_{k}(u)\right)\right)_{h}=\xi \frac{1}{h} \int_{t}^{t+h} \psi\left(T_{k}(u)\right)(s, x) \mathrm{d} s,
$$


where $\xi, \psi$ have the properties stated above. Using [5, Lemma 2.1], we have

$$
\begin{gathered}
\liminf _{h \rightarrow 0}\left\{-\int_{Q}\left(T_{k}(u)-T_{k}\left(u_{0}\right)\right)\left(\xi \frac{1}{h} \int_{t}^{t+h} \psi\left(T_{k}(u)\right)(s, x) \mathrm{d} s\right)_{t} \mathrm{~d} x \mathrm{~d} t\right\} \\
\geq-\int_{Q} \xi_{t} \int_{0}^{T_{k}(u)} \psi(r) \mathrm{d} r \mathrm{~d} x \mathrm{~d} t-\int_{\Omega} \xi(0, x) \int_{0}^{T_{k}\left(u_{0}\right)} \psi(r) \mathrm{d} r \mathrm{~d} x .
\end{gathered}
$$

Therefore, we get from (4.5)

$$
\begin{aligned}
& -\int_{Q} \xi_{t} \int_{0}^{T_{k}(u)} \psi(r) \mathrm{d} r \mathrm{~d} x \mathrm{~d} t-\int_{\Omega} \xi(0, x) \int_{0}^{T_{k}\left(u_{0}\right)} \psi(r) \mathrm{d} r \mathrm{~d} x \\
& \quad+\int_{Q} a\left(t, x, \nabla T_{k}(u)\right) \nabla\left(\xi\left(\psi\left(T_{k}(u)\right)\right)_{h}\right) \mathrm{d} x \mathrm{~d} t \\
& \leq \int_{Q} \xi\left(\psi\left(T_{k}(u)\right)\right)_{h} \mathrm{~d} \mu+\int_{Q} \xi\left(\psi\left(T_{k}(u)\right)\right)_{h} \mathrm{~d} \lambda_{k}+o(1)_{h} .
\end{aligned}
$$

In the energy term, we can let $h$ go to zero since the averages are continuous in $L^{p}\left(0, T ; W_{0}^{1, p}(\Omega)\right)$. We also use that $\left|\left(\psi\left(T_{k}(u)\right)\right)_{h}\right| \leq\|\psi\|_{\infty}$ cap-quasi everywhere in the term with $\lambda_{k}$, and we obtain (4.7). The proof of (4.8) is identical using now [5, Lemma 2.3] for the time derivative.

We immediately deduce the following

COROLLARY 4.7. If $u$ is a renormalized solution of (4.1), then

$$
\begin{aligned}
& \int_{\Omega} \int_{0}^{u(\tau)} \psi(r) \mathrm{d} r \mathrm{~d} x+\int_{0}^{\tau} \int_{\Omega} a(t, x, \nabla u) \nabla u \psi^{\prime}(u) \mathrm{d} x \mathrm{~d} t \\
& \quad \leq \int_{\Omega} \int_{0}^{u_{0}} \psi(r) \mathrm{d} r \mathrm{~d} x+\|\psi\|_{\infty}\|\mu\|_{\mathcal{M}(Q)} \text { for a.e. } \tau \in(0, T),
\end{aligned}
$$

for every nondecreasing $\psi \in W^{1, \infty}(\mathbb{R})$ such that $\psi(0)=0$ and $\psi^{\prime}$ has compact support.

Proof. We use (4.7), where we take $\xi=\xi(t)$, with $0 \leq \xi \leq 1$. First we estimate in an obvious way the term with $\mu$. Then, we let $h \rightarrow 0$. Finally, since $\psi^{\prime}$ has compact support, it is also possible to let $k \rightarrow \infty$, using (4.2). With a standard choice of $\xi$ (e.g., a smooth approximation of $\left.\chi_{(0, \tau)}\right)$ we conclude.

REMARK 4.2. When $\mu \in L^{1}(Q)$, one can pass to the limit in (4.7) and (4.8) when $h \rightarrow 0$ using the continuity of Steklov approximations in $L^{1}$ and the fact that $\psi$ is bounded. Choosing $\psi^{\prime}$ with compact support also allows one to let $k \rightarrow \infty$ and obtain

$$
\begin{aligned}
& -\int_{Q} \Psi(u) \xi_{t} \mathrm{~d} x \mathrm{~d} t-\int_{\Omega} \Psi\left(u_{0}\right) \xi(0, x) \mathrm{d} x+\int_{Q} a(t, x, \nabla u) \nabla(\psi(u) \xi) \mathrm{d} x \mathrm{~d} t \\
& \quad=\int_{Q} \psi(u) \xi \mu \mathrm{d} x \mathrm{~d} t .
\end{aligned}
$$


Of course, one can replace here $\psi$ with $-\psi$ and $\xi$ with $-\xi$; hence, the equality will be true for any Lipschitz function $\psi$ and any $\xi$. We then recover the usual renormalized formulation in case of $L^{1}$-data. The asymptotic estimate for the energy will also be proved later (see (4.26) in Proposition 4.9 below), and this proves that this formulation is equivalent to the one given in [4] for $L^{1}$-data.

We are now able to show that any renormalized solution satisfies the usual estimates and is, in particular, a distributional solution. To this purpose, we only need to precise what we mean by $\nabla u$ when $u$ need not belong to any Sobolev space. We follow the definition of generalized gradient introduced in [2] for functions $u$ whose truncations belong to a Sobolev space:

DEFINITION 4.3. Let $u: Q \rightarrow \mathbb{R}$ be a measurable function which is almost everywhere finite and such that $T_{k}(u) \in L^{p}\left(0, T ; W_{0}^{1, p}(\Omega)\right)$ for every $k>0$. Then (see [2, Lemma 2.1]) there exists a unique vector-valued function $U$ such that

$$
U=\nabla T_{k}(u) \chi_{\{|u|<k\}} \text { a.e. in } Q, \quad \forall k>0 .
$$

This function $U$ will be called the gradient of $u$, hereafter denoted by $\nabla u$. When $u \in L^{1}\left(0, T ; W_{0}^{1,1}(\Omega)\right)$, it coincides with the usual distributional gradient.

We recall the definition of a distributional solution of (4.1). Notice that such a definition makes sense for any measure $\mu$, not necessarily diffuse, even if in our context, we are always dealing with diffuse measures.

DEFINITION 4.4. A function $u \in L^{1}(Q)$ is a distributional solution of problem (4.1) if $T_{k}(u) \in L^{p}\left(0, T ; W_{0}^{1, p}(\Omega)\right)$ for every $k>0$, if $|\nabla u|^{p-1} \in L^{1}(Q)$, and if

$$
-\int_{Q} u \varphi_{t} \mathrm{~d} x \mathrm{~d} t+\int_{Q} a(t, x, \nabla u) \cdot \nabla \varphi \mathrm{d} x \mathrm{~d} t=\int_{Q} \varphi \mathrm{d} \mu+\int_{\Omega} u_{0} \varphi(0, x) \mathrm{d} x,
$$

for any $\varphi \in C_{c}^{\infty}([0, T) \times \Omega)$.

We then have

PROPOSITION 4.5. If $u$ is a renormalized solution of (4.1), then for every $k>0$ and $\tau \leq T$,

$$
\int_{\Omega} \Theta_{k}(u)(\tau) \mathrm{d} x+\int_{0}^{\tau} \int_{\Omega}\left|\nabla T_{k}(u)\right|^{p} \mathrm{~d} x \mathrm{~d} t \leq C k\left(\|\mu\|_{\mathcal{M}(Q)}+\left\|u_{0}\right\|_{L^{1}(\Omega)}\right),
$$

where $\Theta_{k}(s)=\int_{0}^{s} T_{k}(t) \mathrm{d} t$. Therefore,

$$
u \in L^{\infty}\left(0, T ; L^{1}(\Omega)\right)
$$

and

$$
|\nabla u|^{p-1} \in L^{r}(Q) \text { and } a(t, x, \nabla u) \in L^{r}(Q)
$$

for any $r<\frac{N+p^{\prime}}{N+1}$. Moreover, $u$ is a distributional solution. 
Proof. Estimate (4.10) immediately follows from Corollary 4.7 taking $\psi=T_{k}$ and using assumption (2.19). When $k=1$ one deduces that $u \in L^{\infty}\left(0, T ; L^{1}(\Omega)\right)$. Following the results in [6,7], one also deduces the regularity for $|\nabla u|^{p-1}$, hence for $a(t, x, \nabla u)$ in view of (2.20). In particular, we have $a(t, x, \nabla u) \in L^{1}(Q)$, and letting $k \rightarrow+\infty$ in (4.3), we obtain (4.9), i.e., $u$ is a distributional solution.

REMARK 4.3. When $\mu \in \mathcal{M}(Q) \cap L^{p^{\prime}}\left(0, T ; W^{-1, p^{\prime}}(\Omega)\right)$ and $u_{0} \in L^{2}(\Omega)$, there exists a solution of (4.1) in the usual weak sense (see [17]). Such a solution is also a renormalized solution. Indeed, in this case $u \in W$ and satisfies the capacitary estimates of Theorem 2.2. Proceeding like in the proof of Theorem 1.1, one obtains the renormalized formulation for $u$.

We will now prove that the problem (4.1) is well-posed in the class of renormalized solutions. Thanks to the robustness of the formulation, the easiest part here is the uniqueness, which comes from the following comparison principle.

THEOREM 4.8. Let $u_{1}, u_{2}$ be two renormalized solutions of problem (4.1) with data $\left(u_{01}, \mu_{1}\right)$ and $\left(u_{02}, \mu_{2}\right)$ respectively. Then we have

$$
\int_{\Omega}\left(u_{1}-u_{2}\right)^{+}(t) \mathrm{d} x \leq\left\|\left(u_{01}-u_{02}\right)^{+}\right\|_{L^{1}(\Omega)}+\left\|\left(\mu_{1}-\mu_{2}\right)^{+}\right\|_{\mathcal{M}(Q)}
$$

for almost every $t \in(0, T)$. In particular, if $u_{01} \leq u_{02}$ and $\mu_{1} \leq \mu_{2}$ (in the sense of measures), we have $u_{1} \leq u_{2}$ a.e. in $Q$. As a consequence, there exists at most one renormalized solution of problem (4.1).

Proof. Let $\lambda_{k, 1}, \lambda_{k, 2}$ be the measures given by Definition 4.1 corresponding to $u_{1}, u_{2}$. Proposition 4.2 implies

$$
\begin{aligned}
& -\int_{Q}\left(T_{k}\left(u_{1}\right)-T_{k}\left(u_{2}\right)\right) v_{t} \mathrm{~d} x \mathrm{~d} t+\int_{Q}\left(a\left(t, x, \nabla T_{k}\left(u_{1}\right)\right)-a\left(t, x, \nabla T_{k}\left(u_{2}\right)\right)\right) \nabla v \mathrm{~d} x \mathrm{~d} t \\
& =\int_{Q} v \mathrm{~d}\left(\mu_{1}-\mu_{2}\right)+\int_{Q} v \mathrm{~d} \lambda_{k, 1}-\int_{Q} v \mathrm{~d} \lambda_{k, 2}+\int_{\Omega}\left(T_{k}\left(u_{01}\right)-T_{k}\left(u_{02}\right)\right) v(0, x) \mathrm{d} x
\end{aligned}
$$

for every $v \in W \cap L^{\infty}(Q)$ such that $v(T)=0$. Consider the function

$$
w_{h}(t, x)=\frac{1}{h} \int_{t}^{t+h} \frac{1}{\varepsilon} T_{\varepsilon}\left(T_{k}\left(u_{1}\right)-T_{k}\left(u_{2}\right)\right)^{+}(s, x) \mathrm{d} s,
$$

Given $\xi \in C_{c}^{\infty}([0, T)), \xi \geq 0$, take $v=w_{h} \xi$ as test function. Observe that both $w_{h}$ and $\left(w_{h}\right)_{t}$ belong to $L^{p}(0, T ; V) \cap L^{\infty}(Q)$ for $h>0$ sufficiently small, hence $w_{h} \in W \cap L^{\infty}(Q)$. Moreover, we have

$$
w_{h} \rightarrow \frac{1}{\varepsilon} T_{\varepsilon}\left(T_{k}\left(u_{1}\right)-T_{k}\left(u_{2}\right)\right)^{+} \quad \text { strongly in } L^{p}\left(0, T ; W_{0}^{1, p}(\Omega)\right) .
$$


Using that $0 \leq w_{h} \leq 1$ almost everywhere, hence $0 \leq w_{h} \leq 1$ cap-quasi everywhere, we have

$$
\begin{gathered}
-\int_{Q}\left[\left(T_{k}\left(u_{1}\right)-T_{k}\left(u_{2}\right)\right)-\left(T_{k}\left(u_{01}\right)-T_{k}\left(u_{02}\right)\right)\right]\left(w_{h} \xi\right)_{t} \mathrm{~d} x \mathrm{~d} t \\
\quad+\int_{Q}\left(a\left(t, x, \nabla T_{k}\left(u_{1}\right)\right)-a\left(t, x, \nabla T_{k}\left(u_{2}\right)\right)\right) \nabla w_{h} \xi \mathrm{d} x \mathrm{~d} t \\
\leq\|\xi\|_{\infty}\left(\left\|\left(\mu_{1}-\mu_{2}\right)^{+}\right\|_{\mathcal{M}(Q)}+\left\|\lambda_{k, 1}\right\|_{\mathcal{M}(Q)}+\left\|\lambda_{k, 2}\right\|_{\mathcal{M}(Q)}\right) .
\end{gathered}
$$

Using the monotonicity of $T_{\varepsilon}(s)$, we have (see [5, Lemma 2.1])

$$
\begin{aligned}
& \liminf _{h \rightarrow 0}\left\{-\int_{Q}\left[\left(T_{k}\left(u_{1}\right)-T_{k}\left(u_{2}\right)\right)-\left(T_{k}\left(u_{01}\right)-T_{k}\left(u_{02}\right)\right)\right]\left(w_{h} \xi\right)_{t} \mathrm{~d} x \mathrm{~d} t\right\} \\
& \geq-\int_{Q} \hat{\Theta}_{\varepsilon}\left(T_{k}\left(u_{1}\right)-T_{k}\left(u_{2}\right)\right) \xi_{t} \mathrm{~d} x \mathrm{~d} t-\int_{\Omega} \hat{\Theta}_{\varepsilon}\left(T_{k}\left(u_{01}\right)-T_{k}\left(u_{02}\right)\right) \xi(0) \mathrm{d} x
\end{aligned}
$$

where $\hat{\Theta}_{\varepsilon}(s)=\int_{0}^{s} \frac{1}{\varepsilon} T_{\varepsilon}(r)^{+} \mathrm{d} r$. Therefore, letting $h \rightarrow 0$ in (4.12), we obtain

$$
\begin{aligned}
& -\int_{Q} \hat{\Theta}_{\varepsilon}\left(T_{k}\left(u_{1}\right)-T_{k}\left(u_{2}\right)\right) \xi_{t} \mathrm{~d} x \mathrm{~d} t \\
& \quad+\frac{1}{\varepsilon} \int_{Q}\left(a\left(t, x, \nabla T_{k}\left(u_{1}\right)\right)-a\left(t, x, \nabla T_{k}\left(u_{2}\right)\right)\right) \nabla T_{\varepsilon}\left(T_{k}\left(u_{1}\right)-T_{k}\left(u_{2}\right)\right)^{+} \xi \mathrm{d} x \mathrm{~d} t \\
& \leq \int_{\Omega} \hat{\Theta}_{\varepsilon}\left(T_{k}\left(u_{01}\right)-T_{k}\left(u_{02}\right)\right) \xi(0) \mathrm{d} x \\
& \quad+\|\xi\|_{\infty}\left(\left\|\left(\mu_{1}-\mu_{2}\right)^{+}\right\|_{\mathcal{M}(Q)}+\left\|\lambda_{k, 1}\right\|_{\mathcal{M}(Q)}+\left\|\lambda_{k, 2}\right\|_{\mathcal{M}(Q)}\right) .
\end{aligned}
$$

Using (2.21) and letting $\varepsilon \rightarrow 0$, we deduce

$$
\begin{aligned}
& -\int_{Q}\left(T_{k}\left(u_{1}\right)-T_{k}\left(u_{2}\right)\right)^{+} \xi_{t} \mathrm{~d} x \mathrm{~d} t \leq \int_{\Omega}\left(T_{k}\left(u_{01}\right)-T_{k}\left(u_{02}\right)\right)^{+} \xi(0) \mathrm{d} x \\
& \quad+\|\xi\|_{\infty}\left(\left\|\left(\mu_{1}-\mu_{2}\right)^{+}\right\|_{\mathcal{M}(Q)}+\left\|\lambda_{k, 1}\right\|_{\mathcal{M}(Q)}+\left\|\lambda_{k, 2}\right\|_{\mathcal{M}(Q)}\right)
\end{aligned}
$$

and letting $k \rightarrow \infty$, we obtain, thanks to (4.2),

$$
-\int_{Q}\left(u_{1}-u_{2}\right)^{+} \xi_{t} \mathrm{~d} x \mathrm{~d} t \leq\|\xi\|_{\infty}\left(\left\|\left(u_{01}-u_{02}\right)^{+}\right\|_{L^{1}(\Omega)}+\left\|\left(\mu_{1}-\mu_{2}\right)^{+}\right\|_{\mathcal{M}(Q)}\right)
$$

for every nonnegative $\xi \in C_{c}^{\infty}[0, T)$. Of course, the same inequality holds for any $\xi \in W^{1, \infty}(0, T)$ with compact support in $[0, T)$. Take then $\xi(t)=1-\frac{1}{\varepsilon} T_{\varepsilon}(t-\tau)^{+}$, where $\tau \in(0, T)$; since $u_{1}, u_{2} \in L^{\infty}\left(0, T ; L^{1}(\Omega)\right)$, by letting $\varepsilon \rightarrow 0$, we have

$$
-\int_{Q}\left(u_{1}-u_{2}\right)^{+} \xi_{t} \mathrm{~d} x \mathrm{~d} t=\frac{1}{\varepsilon} \int_{\tau}^{\tau+\varepsilon} \int_{\Omega}\left(u_{1}-u_{2}\right)^{+} \mathrm{d} x \mathrm{~d} t \rightarrow \int_{\Omega}\left(u_{1}-u_{2}\right)^{+}(\tau) \mathrm{d} x
$$

for almost every $\tau \in(0, T)$. Using in the right-hand side that $\|\xi\|_{\infty} \leq 1$, we get (4.11). 
REMARK 4.4. The above result continues to hold if we replace condition (2.21) with the more general

$$
[a(t, x, \xi)-a(t, x, \eta)] \cdot(\xi-\eta) \geq 0 \quad \forall \xi, \eta \in \mathbb{R}^{N} .
$$

REMARK 4.5. If $\mu \in \mathcal{M}(Q) \cap L^{p^{\prime}}\left(0, T ; W^{-1, p^{\prime}}(\Omega)\right)$, then $\mu^{+}$is a diffuse measure (since $\mu$ is diffuse) but we can not infer that $\mu^{+}$belongs to $L^{p^{\prime}}\left(0, T ; W^{-1, p^{\prime}}(\Omega)\right.$ ). However, if $u$ is a standard weak solution corresponding to $\mu$, and $v$ is a renormalized solution corresponding to $\mu^{+}$, one can deduce that $u \leq v$. It is enough to proceed as in the above proof using

$$
(t, x) \longmapsto \xi(t) \frac{1}{h} \int_{t}^{t+h} \frac{1}{\varepsilon} T_{\varepsilon}\left(u-T_{k}(v)\right)^{+}(s, x) \mathrm{d} s
$$

as test function.

We immediately deduce the $L^{1}$-contraction estimate.

COROLLARY 4.9. Let $u_{1}, u_{2}$ be two renormalized solutions of problem (4.1) with data $\left(u_{01}, \mu_{1}\right)$ and $\left(u_{02}, \mu_{2}\right)$ respectively. Then we have

$$
\int_{\Omega}\left|u_{1}-u_{2}\right|(t) \mathrm{d} x \leq\left\|u_{01}-u_{02}\right\|_{L^{1}(\Omega)}+\left\|\mu_{1}-\mu_{2}\right\|_{\mathcal{M}(Q)}
$$

for almost every $t \in(0, T)$.

As usual for nonlinear equations with measure data, we will prove the existence of solutions through approximation of the data $\mu, u_{0}$ with smooth functions. We will need the following proposition that collects some known results in the literature.

PROPOSITION 4.6. Let $\left(u_{n}\right)$ be a sequence of solutions of problem

$$
\begin{cases}\left(u_{n}\right)_{t}-\operatorname{div}\left(a\left(t, x, \nabla u_{n}\right)\right)=\mu_{n} & \text { in } Q, \\ u_{n}=u_{0 n} & \text { on }\{0\} \times \Omega, \\ u_{n}=0 & \text { on }(0, T) \times \partial \Omega,\end{cases}
$$

where $\left(u_{0 n}\right)$ strongly converges to $u_{0}$ in $L^{1}(Q)$ and $\left(\mu_{n}\right)$ is a bounded sequence in $L^{1}(\Omega)$. Then,

$$
\left\|u_{n}\right\|_{L^{\infty}\left(0, T ; L^{1}(\Omega)\right)} \leq C,
$$

and

$$
\int_{Q}\left|\nabla T_{k}\left(u_{n}\right)\right|^{p} \mathrm{~d} x \mathrm{~d} t \leq C k \quad \forall k>0 .
$$

Moreover, there exists a measurable function u such that $T_{k}(u) \in L^{p}\left(0, T ; W_{0}^{1, p}(\Omega)\right)$ for any $k>0, u \in L^{\infty}\left(0, T ; L^{1}(\Omega)\right)$, and, up to a subsequence, we have

$$
\begin{aligned}
& u_{n} \rightarrow u \quad \text { a.e. in } Q \text { and strongly in } L^{1}(Q), \\
& T_{k}\left(u_{n}\right) \rightarrow T_{k}(u) \quad \text { weakly in } L^{p}\left(0, T ; W_{0}^{1, p}(\Omega)\right) \text { and a.e. in } Q, \\
& \nabla u_{n} \rightarrow \nabla u \quad \text { a.e. in } Q . \\
& \left|\nabla u_{n}\right|^{p-2} \nabla u_{n} \rightarrow|\nabla u|^{p-2} \nabla u \quad \text { in } L^{1}(Q) .
\end{aligned}
$$


Proof. Estimates (4.15)-(4.16) are classical since the work [7]. The a.e. convergence of $\left(u_{n}\right)$ can be proved by using properties of truncations and the Marcinkiewicz estimates, see e.g., [4] or [22]. The a.e. convergence of $\left(\nabla u_{n}\right)$ is proved in [6] when $u_{0 n}=0$; however, their proof extends to the case of sequences $\left(u_{0 n}\right)$ converging in $L^{1}$, the only difference being to include the initial condition $u_{0 n}$ in the so-called Landes approximation. This latter technical point can be found e.g., in [22].

In order to prove the existence of a renormalized solution, we consider the convolution

$$
\mu_{n}=\rho_{n} * \mu,
$$

where $\rho_{n}$ is a sequence of standard mollifiers. In this way, we can use the equi-diffusion property (see Proposition 3.3) that plays a crucial role in our approach. We will state the convergence result in a slightly more general form which can be applied in case of lower order terms as well.

PROPOSITION 4.7. Let $\left(u_{0 n}\right)$ be a sequence converging strongly to $u_{0}$ in $L^{1}(\Omega)$, let $\left(f_{n}\right)$ be a sequence of bounded functions converging to $f$ strongly in $L^{1}(Q)$, and let $\mu_{n}=\mu * \rho_{n}$. Let $u_{n}$ be the solution of

$$
\begin{cases}\left(u_{n}\right)_{t}-\operatorname{div}\left(a\left(t, x, \nabla u_{n}\right)\right)=f_{n}+\mu_{n} & \text { in } Q, \\ u_{n}=u_{0 n} & \text { on }\{0\} \times \Omega, \\ u_{n}=0 & \text { on }(0, T) \times \partial \Omega .\end{cases}
$$

Then there exists a function u such that (4.17) holds true up to subsequences and $u$ is a renormalized solution, in the sense of Definition 4.1, corresponding to $f+\mu$.

Proof. We split the proof in four steps:

Step 1. Basic estimates.

Both sequences $\left(\mu_{n}\right)$ and $\left(f_{n}\right)$ are bounded in $L^{1}(Q)$. Therefore, the solutions $u_{n}$ satisfy the estimates recalled in Proposition 4.6 and up to a subsequence (4.17) holds. Moreover, Theorem 1.2 applies and gives the estimate

$$
\operatorname{cap}_{p}\left\{\left|u_{n}\right|>k\right\} \leq C \max \left\{k^{-\frac{1}{p}}, k^{-\frac{1}{p^{\prime}}}\right\} \quad \forall k \geq 1, \quad \forall n \geq 1 .
$$

Finally, for $\delta>0$, consider the function $h_{\delta}(s)=\frac{1}{\delta}\left(T_{k+\delta}(s)-T_{k}(s)\right)$, which is a piecewise linear odd function vanishing for $|s| \leq k$ and constant for $|s|>k+\delta$. Let $\xi_{\varepsilon}(t)=1-\frac{1}{\varepsilon} T_{\varepsilon}(t-T+2 \varepsilon)^{+}$, where $\varepsilon>0$; this function belongs to $W^{1, \infty}(0, T)$ and has compact support in $[0, T)$. By using $h_{\delta}\left(u_{n}\right) \xi_{\varepsilon}$ as test function in (4.18) and letting $\varepsilon \rightarrow 0$, we have

$$
\begin{aligned}
& \frac{1}{\delta} \int_{\left\{k<\left|u_{n}\right|<k+\delta\right\}} a\left(t, x, \nabla u_{n}\right) \nabla u_{n} \mathrm{~d} x \mathrm{~d} t \\
& \quad \leq \int_{Q} h_{\delta}\left(u_{n}\right) \mu_{n} \mathrm{~d} x \mathrm{~d} t+\int_{Q} f_{n} h_{\delta}\left(u_{n}\right) \mathrm{d} x \mathrm{~d} t+\int_{\left\{\left|u_{0 n}\right|>k\right\}}\left|u_{0 n}\right| \mathrm{d} x
\end{aligned}
$$


which implies, in particular,

$$
\begin{aligned}
& \frac{1}{\delta} \int_{\left\{k<\left|u_{n}\right|<k+\delta\right\}} a\left(t, x, \nabla u_{n}\right) \nabla u_{n} \mathrm{~d} x \mathrm{~d} t \\
& \quad \leq \int_{\left\{\left|u_{n}\right|>k\right\}}\left|\mu_{n}\right| \mathrm{d} x \mathrm{~d} t+\int_{\left\{\left|u_{n}\right|>k\right\}}\left|f_{n}\right| \mathrm{d} x \mathrm{~d} t+\int_{\left\{\left|u_{0 n}\right|>k\right\}}\left|u_{0 n}\right| \mathrm{d} x
\end{aligned}
$$

Step 2. Equation satisfied by truncations.

For $\delta>0$ small, consider the functions $S_{k, \delta}$ and $T_{k, \delta}$ given by (2.14) and (2.15), respectively. Recall that $T_{k, \delta}$ converges pointwise to $T_{k}$ as $\delta \rightarrow 0$.

Given $\varphi \in C_{c}^{\infty}(Q)$, multiply the equation solved by $u_{n}$ by $S_{k, \delta}\left(u_{n}\right) \varphi$. We then have

$$
\begin{aligned}
& T_{k, \delta}\left(u_{n}\right)_{t}-\operatorname{div}\left(S_{k, \delta}\left(u_{n}\right) a\left(t, x, \nabla u_{n}\right)\right) \\
& =f_{n}+\mu_{n}+\left(S_{k, \delta}\left(u_{n}\right)-1\right)\left(f_{n}+\mu_{n}\right) \\
& \quad+\frac{1}{\delta} a\left(t, x, \nabla u_{n}\right) \nabla u_{n} \operatorname{sign}\left(u_{n}\right) \chi_{\left\{k<\left|u_{n}\right|<k+\delta\right\}} \quad \operatorname{in} \mathcal{D}^{\prime}(Q) .
\end{aligned}
$$

Let

$$
\Lambda_{n, \delta}^{k}:=\left(S_{k, \delta}\left(u_{n}\right)-1\right)\left(f_{n}+\mu_{n}\right)+\frac{1}{\delta} a\left(t, x, \nabla u_{n}\right) \nabla u_{n} \operatorname{sign}\left(u_{n}\right) \chi_{\left\{k<\left|u_{n}\right|<k+\delta\right\}}
$$

With this notation, we have

$$
\left(T_{k}\left(u_{n}\right)\right)_{t}-\operatorname{div}\left(S_{k, \delta}\left(u_{n}\right) a\left(t, x, \nabla u_{n}\right)\right)=f_{n}+\mu_{n}+\Lambda_{n, \delta}^{k} \text { in } \mathcal{D}^{\prime}(Q) .
$$

Thanks to estimate (4.20), and since $\left|S_{k, \delta}\right| \leq 1$, the functions $\Lambda_{n, \delta}^{k}$ are bounded in $L^{1}(Q)$ uniformly with respect to $\delta$ and $n$. Indeed, to be more precise, (4.20) and the definition of $S_{k, \delta}$ imply the estimate

$$
\begin{aligned}
\left\|\Lambda_{n, \delta}^{k}\right\|_{L^{1}(Q)} & \leq \int_{\left\{\left|u_{n}\right|>k\right\}}\left|f_{n}+\mu_{n}\right| \mathrm{d} x \mathrm{~d} t+\frac{1}{\delta} \int_{\left\{k<\left|u_{n}\right|<k+\delta\right\}} a\left(t, x, \nabla u_{n}\right) \nabla u_{n} \mathrm{~d} x \mathrm{~d} t \\
& \leq 2 \int_{\left\{\left|u_{n}\right|>k\right\}}\left|\mu_{n}\right| \mathrm{d} x \mathrm{~d} t+2 \int_{\left\{\left|u_{n}\right|>k\right\}}\left|f_{n}\right| \mathrm{d} x \mathrm{~d} t+\int_{\left\{\left|u_{0 n}\right|>k\right\}}\left|u_{0 n}\right| \mathrm{d} x
\end{aligned}
$$

Step 3. Limit as $n$ goes to infinity.

Applying Proposition 4.6 to the sequence $\left(u_{n}\right)$, there exists a function $u \in L^{1}(Q)$ such that $T_{k}(u) \in L^{p}\left(0, T ; W_{0}^{1, p}(\Omega)\right)$ for all $k>0$ and, up to a subsequence,

$$
u_{n} \rightarrow u, \quad \nabla u_{n} \rightarrow \nabla u \quad \text { a.e. in } Q .
$$

Let now $\left(\delta_{n}\right)$ be a sequence of positive numbers converging to 0 , as $n \rightarrow \infty$. Using the definition of $S_{k, \delta}(t)$ (see (2.14)), the fact that $\nabla u=0$ a.e. in $\{|u|=k\}$ and $a(t, x, 0)=0$ (a consequence of (2.19)), we deduce that

$$
S_{k, \delta_{n}}\left(u_{n}\right) a\left(t, x, \nabla u_{n}\right) \rightarrow a\left(t, x, \nabla T_{k}(u)\right) \text { a.e. in } Q \text {. }
$$


Since $S_{k, \delta_{n}}\left(u_{n}\right) a\left(t, x, \nabla u_{n}\right)$ is bounded in $L^{p^{\prime}}(Q)$, it also follows that

$$
S_{k, \delta_{n}}\left(u_{n}\right) a\left(t, x, \nabla u_{n}\right) \rightarrow a\left(t, x, \nabla T_{k}(u)\right) \text { weakly in } L^{p^{\prime}}(Q) .
$$

By properties of convolution, clearly we have $\mu_{n} \stackrel{*}{\rightarrow} \mu$ in the weak* topology of measures. Finally, since the sequence $\left(\Lambda_{n, \delta_{n}}^{k}\right)_{n}$ is bounded in $L^{1}(\Omega)$, there exists a bounded measure $\lambda_{k}$ such that, up to a subsequence, $\left(\Lambda_{n, \delta_{n}}^{k}\right)_{n}$ converges to $\lambda_{k}$ in the weak* topology of $C(\bar{Q})^{\prime}$. We deduce from (4.21) that $u$ satisfies

$$
\begin{aligned}
& -\int_{Q} T_{k}(u) \varphi_{t} \mathrm{~d} x \mathrm{~d} t+\int_{Q} a\left(t, x, \nabla T_{k}(u)\right) \nabla \varphi \mathrm{d} x \mathrm{~d} t \\
& \quad=\int_{Q} \varphi f \mathrm{~d} x \mathrm{~d} t+\int_{Q} \varphi d \mu+\int_{Q} \varphi d \lambda_{k}+\int_{\Omega} T_{k}\left(u_{0}\right) \varphi(0, x) \mathrm{d} x,
\end{aligned}
$$

for every $\varphi \in C_{c}^{\infty}([0, T) \times \Omega)$.

Step 4. Estimate on $\lambda_{k}$.

By weak* lower semicontinuity of the norm, we have

$$
\left\|\lambda_{k}\right\|_{\mathcal{M}(Q)} \leq \liminf _{n \rightarrow \infty}\left\|\Lambda_{n, \delta_{n}}^{k}\right\|_{\mathcal{M}(Q)} .
$$

Since, by Proposition 3.3, the sequence $\left(\mu_{n}\right)$ is equidiffuse, thanks to the uniform estimate (4.19), we deduce that

$$
\lim _{k \rightarrow \infty} \sup _{n} \int_{\left\{\left|u_{n}\right|>k\right\}}\left|\mu_{n}\right| \mathrm{d} x \mathrm{~d} t=0 .
$$

By the equi-integrability of $\left(u_{0 n}\right)$ and $\left(f_{n}\right)$, the last two terms in (4.22) satisfy a similar property. Then, by (4.22), we have

$$
\left\|\lambda_{k}\right\|_{\mathcal{M}(Q)} \leq \liminf _{n \rightarrow \infty}\left\|\Lambda_{n, \delta_{n}}^{k}\right\|_{\mathcal{M}(Q)} \leq \varepsilon_{k}
$$

where $\varepsilon_{k}$ is some quantity which tends to zero as $k \rightarrow \infty$. Letting $k \rightarrow \infty$, we conclude that $\lambda_{k}$ satisfies (4.2), and hence, $u$ is a renormalized solution.

We immediately deduce by the previous results the following

THEOREM 4.10. Let $\mu$ be a diffuse measure, and $u_{0} \in L^{1}(\Omega)$. Assume that (2.19)(2.21) hold true. Then there exists a unique renormalized solution of (4.1) in the sense of Definition 4.1.

Let us point out that the same arguments used in the construction of a renormalized solution could also be employed for a stability result of renormalized solutions corresponding to data $\mu$ which are weakly converging and equidiffuse. However, to this purpose, one would need the capacitary estimate of Theorem 1.2 to hold for renormalized solutions as well. A major problem here is that solutions with measure data may not possess a cap-quasi continuous representative; hence, $\operatorname{cap}_{p}(\{|u|>k\})$ is not well defined for all renormalized solutions $u$. To overcome this obstacle, the following weak form of Theorem 1.2 can be proved. 
PROPOSITION 4.8. Let $u$ be a renormalized solution of (4.1). Then, for every $m \geq 1$ there exist positive functions $z_{m}, w_{m} \in W$ such that:

(i) $-w_{m} \leq T_{m}(u) \leq z_{m}$ a.e. in $Q$.

(ii) $\left\|z_{m}\right\|_{W}+\left\|w_{m}\right\|_{W} \leq C \max \left\{m^{\frac{1}{p}}, m^{\frac{1}{p^{\prime}}}\right\}$

where $C=C\left(\|\mu\|_{\mathcal{M}(Q)},\left\|u_{0}\right\|_{L^{1}(\Omega)}, p, \alpha, \beta,\|b\|_{L^{p^{\prime}}(Q)}\right)$. In particular,

$$
\operatorname{cap}_{p}\left(\left\{z_{m}>m\right\}\right)+\operatorname{cap}_{p}\left(\left\{w_{m}>m\right\}\right) \leq C \max \left\{\frac{1}{m^{\frac{1}{p}}}, \frac{1}{m^{\frac{1}{p^{\prime}}}}\right\} .
$$

Proof. The proof is done following that of Theorem 1.2. Let us only precise a few technical modifications. First of all, we start by considering the case where $\mu \geq 0$ and $u_{0} \geq 0$, which implies that $u \geq 0$ by Theorem 4.8. The estimate in Step 1 of Theorem 1.2 follows from Proposition 4.5. Next, we construct the function $z_{m}$ in the same way as in Step 2 of Theorem $1.2\left(\right.$ replacing $-\Delta_{p}(\cdot)$ with $\left.-\operatorname{div}(a(t, x, \nabla(\cdot)))\right)$ : here, we assume that $T_{m}(u(T))$ is well defined (there is however no loss of generality, otherwise one can use some sequence $t_{n} \uparrow T$ such that $T_{m}(u)\left(t_{n}\right)$ is well defined, recall that $u \in L^{\infty}\left(0 ; T ; L^{1}(\Omega)\right)$ and such a sequence of Lebesgue points certainly exists). Using assumptions (2.19) and (2.20), one obtains the estimate on $z_{m}$ :

$$
\left\|z_{m}\right\|_{W} \leq C \max \left\{m^{\frac{1}{p}}, m^{\frac{1}{p^{\prime}}}\right\} \quad \text { where } C=C\left(\|\mu\|,\left\|u_{0}\right\|, p, \alpha, \beta,\|b\|_{L^{p^{\prime}}(Q)}\right) .
$$

Finally, to conclude that $z_{m} \geq T_{m}(u)$, we show that

$$
T_{m}(u)_{t}-\operatorname{div}\left(a\left(t, x, \nabla T_{m}(u)\right)\right) \geq 0 .
$$

To this purpose, we use (4.7) with $\psi=-S_{m, \delta}\left(r^{+}\right)$, where $S_{m, \delta}$ is defined in (2.14). Since $\mu \geq 0$, we have, for every nonnegative $\xi \in C_{c}^{\infty}([0, T) \times \Omega)$,

$$
\int_{Q} \xi \psi\left(T_{k}(u)\right)_{h} \mathrm{~d} \mu+\|\xi\|_{\infty}\|\psi\|_{\infty}\left\|\lambda_{k}\right\| \leq\|\xi\|_{\infty}\left\|\lambda_{k}\right\| \rightarrow 0 .
$$

Then, we let $h \rightarrow 0, k \rightarrow \infty$ and we obtain

$$
\begin{aligned}
& -\int_{Q} \xi_{t} \int_{0}^{u} S_{m, \delta}\left(r^{+}\right) \mathrm{d} r \mathrm{~d} x \mathrm{~d} t-\int_{\Omega} \xi(0, x) \int_{0}^{u_{0}} S_{m, \delta}\left(r^{+}\right) \mathrm{d} r \mathrm{~d} x \\
& \quad+\int_{Q} a(t, x, \nabla u) \nabla\left(S_{m, \delta}\left(u^{+}\right) \xi\right) \mathrm{d} x \mathrm{~d} t \geq 0 .
\end{aligned}
$$

Recalling that $u \geq 0$, dropping the term with $S_{m, \delta}^{\prime}$ which is negative, and letting $\delta \rightarrow 0$, we deduce (4.23). Therefore, we complete Step 3 of Theorem 1.2 and the conclusion in case that $\mu \geq 0$ and $u_{0} \geq 0$. Since $-\mu^{-} \leq \mu \leq \mu^{+}$and $-u_{0}^{-} \leq u_{0} \leq u_{0}^{+}$, the general case can be obtained comparing $u$ with the solutions corresponding to $\mu^{+}, u_{0}^{+}$ and $-\mu^{-},-u_{0}^{-}$, which is possible thanks to the comparison principle for renormalized solutions (Theorem 4.8). 
Let us notice that, in the previous proof, we do not need anymore the regularizing procedure of Step 5 of Theorem 1.2 since we can compare directly renormalized solutions which are defined even if data do not belong to $L^{p^{\prime}}\left(0, T ; W^{-1, p^{\prime}}(\Omega)\right)$.

We deduce now an energy estimate for the renormalized solutions which extends the classical one known in case of $L^{1}$ data.

PROPOSITION 4.9. Let $u$ be a renormalized solution of (4.1). Then, for every $m \geq 1$ there exists a cap-quasi open set $E_{m} \subset Q$ such that

$$
\operatorname{cap}_{p}\left(E_{m}\right) \leq C \max \left\{\frac{1}{m^{\frac{1}{p}}}, \frac{1}{m^{\frac{1}{p^{\prime}}}}\right\}
$$

where $C=C\left(\|\mu\|_{\mathcal{M}(Q)},\left\|u_{0}\right\|_{L^{1}(\Omega)}, p, \alpha, \beta,\|b\|_{L^{p^{\prime}}(Q)}\right)$, and, for every $\delta \in(0,1]$,

$$
\frac{1}{\delta} \int_{\{m<|u|<m+\delta\}} a(t, x, \nabla u) \nabla u \mathrm{~d} x \mathrm{~d} t \leq \int_{\left\{\left|u_{0}\right|>m\right\}}\left|u_{0}\right| \mathrm{d} x+|\mu|\left(E_{m}\right) .
$$

In particular, we have

$$
\lim _{m \rightarrow \infty} \int_{\{m<|u|<m+1\}} a(t, x, \nabla u) \nabla u \mathrm{~d} x \mathrm{~d} t=0 .
$$

Moreover, we have the estimate

$$
\left\|\lambda_{m}\right\|_{\mathcal{M}(Q)} \leq \int_{\left\{\left|u_{0}\right|>m\right\}}\left|u_{0}\right| \mathrm{d} x+2|\mu|\left(E_{m}\right)
$$

where $\lambda_{m}$ is the measure associated to the renormalized equation of $u$.

Proof. Let $\psi_{m, \delta}(s)$ be a $C^{2}(\mathbb{R})$ function which is nondecreasing, odd and such that $\psi_{m, \delta}(s)=0$ if $|s| \leq m$ and $\psi_{m, \delta}(s)=1$ if $s \geq m+\delta$. We use (4.7) with $\xi \in$ $C_{c}^{\infty}([0, T))$ and $\psi=\psi_{m, \delta}$. Assuming $k>m+1$, we obtain

$$
\begin{aligned}
& -\int_{Q} \xi_{t} \int_{0}^{T_{k}(u)} \psi_{m, \delta}(r) \mathrm{d} r \mathrm{~d} x \mathrm{~d} t+\int_{\{m<|u|<m+\delta\}} a(t, x, \nabla u) \nabla u \psi_{m, \delta}^{\prime}(u) \xi \mathrm{d} x \mathrm{~d} t \\
& \quad \leq \int_{Q} \xi\left(\psi_{m, \delta}\left(T_{k}(u)\right)\right)_{h} \mathrm{~d} \mu+\|\xi\|_{\infty}\left\|\lambda_{k}\right\|_{\mathcal{M}(Q)}+\|\xi\|_{\infty} \int_{\left\{\left|u_{0}\right|>m\right\}}\left|u_{0}\right| \mathrm{d} x+o(1)_{h}
\end{aligned}
$$

where $o(1)_{h}$ tends to zero as $h \rightarrow 0$. Taking $\xi_{\varepsilon} \in C_{c}^{\infty}([0, T))$ such that $\left(\xi_{\varepsilon}\right)_{t} \leq 0$ and $\xi_{\varepsilon} \nearrow 1$ pointwise in $(0, T)$, we get

$$
\begin{aligned}
& \int_{\{m<|u|<m+\delta\}} a(t, x, \nabla u) \nabla u \psi_{m, \delta}^{\prime}(u) \mathrm{d} x \mathrm{~d} t \\
& \quad \leq \int_{Q}\left|\psi_{m, \delta}\left(T_{k}(u)\right)_{h}\right| \mathrm{d}|\mu|+\left\|\lambda_{k}\right\|_{\mathcal{M}(Q)}+\int_{\left\{\left|u_{0}\right|>m\right\}}\left|u_{0}\right| \mathrm{d} x+o(1)_{h} .
\end{aligned}
$$

Since $k>m+1$, we have $\psi_{m, \delta}\left(T_{k}(u)\right)=\psi_{m, \delta}\left(T_{m+1}(u)\right)$; hence, using Proposition 4.8, we have

$$
\psi_{m, \delta}\left(T_{k}(u)\right)^{+} \leq \psi_{m, \delta}\left(z_{m+1}\right) \text { and } \psi_{m, \delta}\left(T_{k}(u)\right)^{-} \leq \psi_{m, \delta}\left(w_{m+1}\right)
$$


and since the inequalities are preserved taking the average and the cap-quasi continuous representatives we deduce from (4.28):

$$
\begin{aligned}
& \int_{\{m<|u|<m+\delta\}} a(t, x, \nabla u) \nabla u \psi_{m, \delta}^{\prime}(u) \mathrm{d} x \mathrm{~d} t \\
& \leq \int_{Q}\left[\left(\psi_{m, \delta}\left(z_{m+1}\right)\right)_{h}+\left(\psi_{m, \delta}\left(w_{m+1}\right)\right)_{h}\right] \mathrm{d}|\mu|+\left\|\lambda_{k}\right\|_{\mathcal{M}(Q)} \\
& \quad+\int_{\left\{\left|u_{0}\right|>m\right\}}\left|u_{0}\right| \mathrm{d} x+o(1)_{h} .
\end{aligned}
$$

Applying Lemma 4.1, for any $z \in W$, we have $\psi_{m, \delta}(z) \in S \cap L^{\infty}(Q)$; hence, it follows from Lemma 4.5 that, up to subsequences, $\left(\psi_{m, \delta}(z)\right)_{h} \rightarrow \psi_{m, \delta}(z)$ capquasi everywhere. We deduce, by dominated convergence, that $\left(\psi_{m, \delta}\left(z_{m+1}\right)\right)_{h}$ and $\left(\psi_{m, \delta}\left(w_{m+1}\right)\right)_{h}$ both converge in $L^{1}(d|\mu|)$. Then, passing to the limit as $h \rightarrow 0$ and then $k \rightarrow \infty$, we obtain

$$
\begin{aligned}
& \int_{\{m<|u|<m+\delta\}}^{a(t, x, \nabla u) \nabla u \psi_{m, \delta}^{\prime}(u) \mathrm{d} x \mathrm{~d} t} \\
& \quad \leq \int_{Q}\left[\left(\psi_{m, \delta}\left(z_{m+1}\right)\right)+\left(\psi_{m, \delta}\left(w_{m+1}\right)\right)\right] \mathrm{d}|\mu|+\int_{\left\{\left|u_{0}\right|>m\right\}}\left|u_{0}\right| \mathrm{d} x
\end{aligned}
$$

Setting $E_{m}=\left\{(t, x): z_{m+1}>m\right\} \cup\left\{(t, x): w_{m+1}>m\right\}$, we have, by definition of $\psi_{m, \delta}$, that

$$
\int_{Q}\left[\left(\psi_{m, \delta}\left(z_{m+1}\right)\right)+\left(\psi_{m, \delta}\left(w_{m+1}\right)\right)\right] \mathrm{d}|\mu| \leq|\mu|\left(E_{m}\right)
$$

hence, we get

$$
\int_{\{m<|u|<m+\delta\}} a(t, x, \nabla u) \nabla u \psi_{m, \delta}^{\prime}(u) \mathrm{d} x \mathrm{~d} t \leq|\mu|\left(E_{m}\right)+\int_{\left\{\left|u_{0}\right|>m\right\}}\left|u_{0}\right| \mathrm{d} x,
$$

for every nondecreasing, odd function $\psi \in C^{2}(\mathbb{R})$ such that $\psi_{m, \delta}=0$ if $|s| \leq m$ and $\psi_{m, \delta}=1$ if $s \geq m+\delta$. By approximations with $C^{2}$ functions, the same inequality will be satisfied by the piecewise linear function $\psi_{m, \delta}=\frac{1}{\delta} T_{\delta}\left(s-T_{m}(s)\right)$; hence, we get (4.25). Since

$$
\operatorname{cap}_{p}\left(E_{m}\right) \leq \frac{\left\|z_{m+1}\right\|_{W}}{m}+\frac{\left\|w_{m+1}\right\|_{W}}{m}
$$

the estimate (4.24) follows from Proposition 4.8. Then, since $\mu$ is diffuse, (4.25) implies (4.26).

Finally, we want to estimate $\left\|\lambda_{m}\right\|_{\mathcal{M}(Q)}$. To this aim, we use Lemma 4.6 and in particular (4.7) with $\psi=\psi_{m, \delta}\left(-s^{-}\right)$and (4.8) with $\psi=\psi_{m, \delta}\left(s^{+}\right)$, again with 
$\psi_{m, \delta} \in C^{2}(\mathbb{R})$ (and with the same properties as above). We subtract the two inequalities, and we find

$$
\begin{aligned}
& -\int_{Q} \xi_{t} \int_{T_{k}\left(u_{0}\right)}^{T_{k}(u)}\left(\psi_{m, \delta}\left(-r^{-}\right)-\psi_{m, \delta}\left(r^{+}\right)\right)(r) \mathrm{d} r \\
& \quad+\int_{Q} a\left(t, x, \nabla T_{k}(u)\right) \nabla\left[\xi\left(\psi_{m, \delta}\left(-T_{k}(u)^{-}\right)-\psi_{m, \delta}\left(T_{k}(u)^{+}\right)\right)\right] \mathrm{d} x \mathrm{~d} t \\
& \quad \leq \int_{Q} \xi \psi_{m, \delta}\left(-T_{k}(u)^{-}\right)_{h} \mathrm{~d} \mu-\int_{Q} \xi \psi_{m, \delta}\left(T_{k}(u)^{+}\right)_{-h} \mathrm{~d} \mu \\
& \quad+2\|\xi\|_{\infty}\left\|\lambda_{k}\right\|_{\mathcal{M}(Q)}+o(1)_{h} .
\end{aligned}
$$

We add (4.3) (with $\varphi=\xi$ ) to the above inequality, and we set $\zeta_{m, \delta}(s)=\psi_{m, \delta}\left(-s^{-}\right)-$ $\psi_{m, \delta}\left(s^{+}\right)+1$. Note that $\zeta_{m, \delta}(s)=-\operatorname{sign}(s) \psi_{m, \delta}(s)+1$ and that $\zeta_{m, \delta} \rightarrow \chi_{|s| \leq m}$ as $\delta \rightarrow 0$. We obtain

$$
\begin{aligned}
& -\int_{Q} \xi_{t} \int_{T_{k}\left(u_{0}\right)}^{T_{k}(u)} \zeta_{m, \delta}(r) \mathrm{d} r \\
& \quad+\int_{Q} a\left(t, x, \nabla T_{k}(u)\right) \nabla\left[\xi\left(\zeta_{m, \delta}\left(T_{k}(u)\right)\right)\right] \mathrm{d} x \mathrm{~d} t-\int_{Q} \xi d \mu-\int_{Q} \xi \mathrm{d} \lambda_{k} \\
& \quad \leq \int_{Q} \xi \psi_{m, \delta}\left(-T_{k}(u)^{-}\right)_{h} \mathrm{~d} \mu-\int_{Q} \xi \psi_{m, \delta}\left(T_{k}(u)^{+}\right)_{-h} \mathrm{~d} \mu \\
& \quad+2\|\xi\|_{\infty}\left\|\lambda_{k}\right\|_{\mathcal{M}(Q)}+o(1)_{h} .
\end{aligned}
$$

Using (4.29) and since $\zeta_{m, \delta}\left(T_{k}(u)\right)=\zeta_{m, \delta}(u)$ for $k>m+1$, we deduce

$$
\begin{aligned}
& -\int_{Q} \xi_{t} \int_{u_{0}}^{u} \zeta_{m, \delta}(r) \mathrm{d} r+\int_{Q} a(t, x, \nabla u) \nabla\left[\xi\left(\zeta_{m, \delta}(u)\right)\right] \mathrm{d} x \mathrm{~d} t-\int_{Q} \xi \mathrm{d} \mu \\
& \leq\|\xi\|_{\infty} \int_{Q}\left[\left(\psi_{m, \delta}\left(z_{m+1}\right)\right)_{-h}+\left(\psi_{m, \delta}\left(w_{m+1}\right)\right)_{h}\right] \mathrm{d}|\mu| \\
& \quad+3\|\xi\|_{\infty}\left\|\lambda_{k}\right\|_{\mathcal{M}(Q)}+o(1)_{h},
\end{aligned}
$$

The right-hand side can be estimated as before, letting $h \rightarrow 0$, obtaining

$$
\begin{aligned}
& -\int_{Q} \xi_{t} \int_{u_{0}}^{u} \zeta_{m, \delta}(r) \mathrm{d} r+\int_{Q} a(t, x, \nabla u) \nabla \xi \zeta_{m, \delta}(u) \mathrm{d} x \mathrm{~d} t-\int_{Q} \xi \mathrm{d} \mu \\
& \quad \leq-\int_{Q} a(t, x, \nabla u) \nabla u \zeta_{m, \delta}^{\prime}(u) \xi \mathrm{d} x \mathrm{~d} t+\|\xi\|_{\infty}|\mu|\left(E_{m}\right)+3\|\xi\|_{\infty}\left\|\lambda_{k}\right\|_{\mathcal{M}(Q)}
\end{aligned}
$$

Now, let $k \rightarrow \infty$ using (4.2). Since $\zeta_{m, \delta}^{\prime}=-\operatorname{sign}(s) \psi_{m, \delta}^{\prime}(s)$, thanks to (4.30), we end up with

$$
\begin{aligned}
& -\int_{Q} \xi_{t} \int_{u_{0}}^{u} \zeta_{m, \delta}(r) \mathrm{d} r+\int_{Q} a(t, x, \nabla u) \nabla \xi \zeta_{m, \delta}(u) \mathrm{d} x \mathrm{~d} t-\int_{Q} \xi \mathrm{d} \mu \\
& \quad \leq\|\xi\|_{\infty} \int_{\left\{\left|u_{0}\right|>m\right\}}\left|u_{0}\right| \mathrm{d} x+2\|\xi\|_{\infty}|\mu|\left(E_{m}\right) .
\end{aligned}
$$


Letting $\delta \rightarrow 0$, we find in the left hand side the term $T_{m}(u)_{t}-\operatorname{div}\left(a\left(t, x, \nabla T_{m}(u)\right)\right)-\mu$ which, using (4.3), coincides with $\lambda_{m}$, hence

$$
\int_{Q} \xi \mathrm{d} \lambda_{m} \leq\|\xi\|_{\infty} \int_{\left\{\left|u_{0}\right|>m\right\}}\left|u_{0}\right| \mathrm{d} x+2\|\xi\|_{\infty}|\mu|\left(E_{m}\right) .
$$

The same inequality can be obtained for $-\lambda_{m}$ using now (4.7) with $\psi=\psi_{m, \delta}\left(s^{+}\right)$ and (4.8) with $\psi=\psi_{m, \delta}\left(-s^{-}\right)$. Finally, we conclude

$$
\left|\int_{Q} \xi \mathrm{d} \lambda_{m}\right| \leq\|\xi\|_{\infty}\left[\int_{\left\{\left|u_{0}\right|>m\right\}}\left|u_{0}\right| \mathrm{d} x+2|\mu|\left(E_{m}\right)\right]
$$

which implies (4.27).

REMARK 4.6. Thanks to Proposition 4.9, it is possible to prove the stability of renormalized solutions with respect to a sequence of data $\left(\mu_{n}\right)$ which are weakly converging (in the sense of measures) and equidiffuse. In particular, the estimate (4.27) implies that the condition (4.2) on the sequence $\left(\lambda_{k}\right)$ holds uniformly (hence it is stable) when the measures $\mu_{n}$ are equidiffuse.

Finally, we conclude this section by showing that Definition 4.1 implies that $u$ is a renormalized solution in the sense of [13]. Since both solutions have been proved to be unique, in particular, this proves that the formulations are actually equivalent.

THEOREM 4.11. Let $u$ be a renormalized solution according to Definition 4.1, and let $\mu$ be split as in (1.5), namely

$$
\mu=f-\operatorname{div}(G)+g_{t}, \quad f \in L^{1}(\Omega), G \in L^{p^{\prime}}(Q), g \in L^{p}(0, T ; V) .
$$

Then u satisfies:

$$
\begin{aligned}
& u-g \in L^{\infty}\left(0, T ; L^{1}(\Omega)\right), \quad T_{k}(u-g) \in L^{p}\left(0, T ; W_{0}^{1, p}(\Omega)\right) \quad \forall k>0, \\
& \lim _{h \rightarrow \infty} \int_{\{h<|u-g|<h+1\}}|\nabla u|^{p} \mathrm{~d} x \mathrm{~d} t=0, \\
& -\int_{Q} S(u-g) \varphi_{t} \mathrm{~d} x \mathrm{~d} t+\int_{Q} a(t, x, \nabla u) \nabla\left(S^{\prime}(u-g) \varphi\right) \mathrm{d} x \mathrm{~d} t \\
& =\int_{Q} f S^{\prime}(u-g) \varphi \mathrm{d} x \mathrm{~d} t+\int_{Q} G \nabla\left(S^{\prime}(u-g) \varphi\right) \mathrm{d} x \mathrm{~d} t+\int_{\Omega} S\left(u_{0}\right) \varphi(0, x) \mathrm{d} x
\end{aligned}
$$

for every $S \in W^{2, \infty}(\mathbb{R})$ such that $S^{\prime}$ has compact support, for every $\varphi \in C_{c}^{\infty}([0, T)$ $\times \Omega$ ).

Proof. We split the proof in two steps.

Step 1. Set $v=T_{k}(u)-g$. Then $v \in L^{p}(0, T ; V)$. Moreover, using the decomposition of $\mu$ in (4.3), and integrating by parts the term with $g_{t}$, we see that $v$ satisfies

$$
\begin{aligned}
& -\int_{Q} v \varphi_{t} \mathrm{~d} x \mathrm{~d} t+\int_{Q} a\left(t, x, \nabla T_{k}(u)\right) \nabla \varphi d x d t \\
& \quad=\int_{Q} f \varphi \mathrm{d} x \mathrm{~d} t+\int_{Q} G \nabla \varphi \mathrm{d} x \mathrm{~d} t+\int_{Q} \varphi d \lambda_{k}+\int_{\Omega} T_{k}\left(u_{0}\right) \varphi(0, x) \mathrm{d} x
\end{aligned}
$$


for every $\varphi \in C_{c}^{\infty}([0, T) \times \Omega)$. It is easy to see that the above equality remains true for every $\varphi \in W^{1, \infty}(Q)$. Take then

$$
\varphi(x, t)=\xi(x, t) \frac{1}{h} \int_{t}^{t+h} \psi(v(s, x)) \mathrm{d} s,
$$

where $\xi \in C_{c}^{\infty}([0, T) \times \bar{\Omega}), \xi \geq 0, \xi \psi(0)=0$ on $(0, T) \times \partial \Omega$, and $\psi$ is a Lipschitz nondecreasing function. Since $\psi$ is nondecreasing, we have (using [5, Lemma 2.1])

$$
\begin{aligned}
& \liminf _{h \rightarrow 0}\left\{-\int_{Q}\left(v-T_{k}\left(u_{0}\right)\right)\left(\xi \frac{1}{h} \int_{t}^{t+h} \psi(v) d s\right)_{t} \mathrm{~d} x \mathrm{~d} t\right\} \\
& \geq-\int_{Q}\left(\int_{0}^{v} \psi(r) d r\right) \xi_{t} \mathrm{~d} x \mathrm{~d} t-\int_{\Omega}\left(\int_{0}^{T_{k}\left(u_{0}\right)} \psi(r) d r\right) \xi(0, x) \mathrm{d} x .
\end{aligned}
$$

Moreover, since $\psi$ is bounded, we have

$$
\left|\int_{Q} \varphi \mathrm{d} \lambda_{k}\right| \leq\|\xi\|_{\infty}\|\psi\|_{\infty}\left\|\lambda_{k}\right\|_{\mathcal{M}(Q)},
$$

and since $\psi$ is Lipschitz, we have $\psi(v) \in L^{p}\left(0, T ; W_{0}^{1, p}(\Omega)\right)$; hence, $(\psi(v))_{h}$ converges to $\psi(v)$ strongly in $L^{p}\left(0, T ; W_{0}^{1, p}(\Omega)\right)$ and weakly* in $L^{\infty}(Q)$. Therefore, we deduce, as $h \rightarrow 0$,

$$
\begin{aligned}
& -\int_{Q}\left(\int_{0}^{v} \psi(r) d r\right) \xi \mathrm{d} x \mathrm{~d} t+\int_{Q} a\left(t, x, \nabla T_{k}(u)\right) \nabla(\psi(v) \xi) \mathrm{d} x \mathrm{~d} t \\
& \leq \int_{Q} f \psi(v) \xi \mathrm{d} x \mathrm{~d} t+\int_{Q} G \nabla(\psi(v) \xi) \mathrm{d} x \mathrm{~d} t \\
& \quad+\int_{\Omega}\left(\int_{0}^{T_{k}\left(u_{0}\right)} \psi(r) \mathrm{d} r\right) \xi(0, x) \mathrm{d} x+\|\xi\|_{\infty}\|\psi\|_{\infty}\left\|\lambda_{k}\right\|_{\mathcal{M}(Q)},
\end{aligned}
$$

for every $\psi$ Lipschitz and nondecreasing. In order to obtain the reverse inequality, one can take

$$
\varphi(x, t)=\xi(x, t) \frac{1}{h} \int_{t-h}^{t} \psi(\hat{v}(s, x)) \mathrm{d} s
$$

where $\hat{v}(x, t)=v(x, t)$ when $t \geq 0$ and $\hat{v}=U_{j}$ when $t<0$, being $U_{j} \in C_{c}^{\infty}(\Omega)$ such that $U_{j} \rightarrow T_{k}\left(u_{0}\right)$ strongly in $L^{1}(\Omega)$. Using this time [5, Lemma 2.3], we obtain

$$
\begin{aligned}
\liminf _{h \rightarrow 0}\left\{-\int_{Q}\left(v-T_{k}\left(u_{0}\right)\right)\left(\xi \frac{1}{h} \int_{t-h}^{t} \psi(v) d s\right)_{t} \mathrm{~d} x \mathrm{~d} t\right\} \\
\leq-\int_{Q}\left(\int_{0}^{v} \psi(r) d r\right) \xi t \mathrm{~d} x \mathrm{~d} t-\int_{\Omega}\left(\int_{0}^{U_{j}} \psi(r) d r\right) \xi(0, x) \mathrm{d} x \\
\quad-\int_{\Omega}\left(T_{k}\left(u_{0}\right)-U_{j}\right) \psi\left(U_{j}\right) \xi(0, x) \mathrm{d} x .
\end{aligned}
$$


Since it is still true that $\hat{v} \in L^{p}\left(0, T ; W_{0}^{1, p}(\Omega)\right) \cap L^{\infty}(Q)$, when $h \rightarrow 0$, we can pass to the limit in the other terms as above, and we obtain now

$$
\begin{aligned}
& -\int_{Q}\left(\int_{0}^{v} \psi(r) d r\right) \xi_{t} \mathrm{~d} x \mathrm{~d} t+\int_{Q} a\left(t, x, \nabla T_{k}(u)\right) \nabla(\psi(v) \xi) \mathrm{d} x \mathrm{~d} t \\
& \geq \int_{Q} f \psi(v) \xi \mathrm{d} x \mathrm{~d} t+\int_{Q} G \nabla(\psi(v) \xi) \mathrm{d} x \mathrm{~d} t+\int_{\Omega}\left(\int_{0}^{U_{j}} \psi(r) d r\right) \xi(0, x) \mathrm{d} x \\
& \quad+\int_{\Omega}\left(T_{k}\left(u_{0}\right)-U_{j}\right) \psi\left(U_{j}\right) \xi(0, x) \mathrm{d} x-\|\xi\|_{\infty}\|\psi\|_{\infty}\left\|\lambda_{k}\right\|_{\mathcal{M}(Q)},
\end{aligned}
$$

which implies, as $U_{j} \rightarrow T_{k}\left(u_{0}\right)$, that

$$
\begin{aligned}
& -\int_{Q}\left(\int_{0}^{v} \psi(r) d r\right) \xi_{t} \mathrm{~d} x \mathrm{~d} t+\int_{Q} a\left(t, x, \nabla T_{k}(u)\right) \nabla(\psi(v) \xi) \mathrm{d} x \mathrm{~d} t \\
& \geq \int_{Q} f \psi(v) \xi \mathrm{d} x \mathrm{~d} t+\int_{Q} G \nabla(\psi(v) \xi) \mathrm{d} x \mathrm{~d} t+\int_{\Omega}\left(\int_{0}^{T_{k}\left(u_{0}\right)} \psi(r) d r\right) \xi(0, x) \mathrm{d} x \\
& \quad-\|\xi\|_{\infty}\|\psi\|_{\infty}\left\|\lambda_{k}\right\|_{\mathcal{M}(Q) .}
\end{aligned}
$$

Let $S \in W^{2, \infty}(\mathbb{R})$; we use (4.34) with $\psi=\int_{0}^{s}\left(S^{\prime \prime}(t)\right)^{+} \mathrm{d} t$ and (4.35) with $\psi=\int_{0}^{s}\left(S^{\prime \prime}(t)\right)^{-} \mathrm{d} t$. Since $S^{\prime}(s)=\int_{0}^{s}\left(S^{\prime \prime}(t)^{+}-S^{\prime \prime}(t)^{-}\right) \mathrm{d} t$, subtracting the two inequalities, we obtain

$$
\begin{aligned}
& -\int_{Q} S(v) \xi_{t} \mathrm{~d} x \mathrm{~d} t+\int_{Q} a\left(t, x, \nabla T_{k}(u)\right) \nabla\left(S^{\prime}(v) \xi\right) \mathrm{d} x \mathrm{~d} t \\
& \leq \int_{Q} f S^{\prime}(v) \xi \mathrm{d} x \mathrm{~d} t+\int_{Q} G \nabla\left(S^{\prime}(v) \xi\right) \mathrm{d} x \mathrm{~d} t \\
& \quad+\int_{\Omega} S\left(T_{k}\left(u_{0}\right)\right) \xi(0, x) \mathrm{d} x+2\|\xi\|_{\infty}\left\|S^{\prime}\right\|_{\infty}\left\|\lambda_{k}\right\|_{\mathcal{M}(Q)},
\end{aligned}
$$

for every $S \in W^{2, \infty}(\mathbb{R})$ and for every nonnegative $\xi$.

Step 2. Take in (4.36) $S^{\prime}=\theta_{h}(s)$, where $\theta_{h}=T_{1}\left(s-T_{h}(s)\right)$ and $\xi=\xi(t)$. We obtain, denoting $R_{h}(s)=\int_{0}^{s} \theta_{h}(\xi) \mathrm{d} \xi$,

$$
\begin{aligned}
& -\int_{Q} R_{h}\left(T_{k}(u)-g\right) \xi_{t} \mathrm{~d} x \mathrm{~d} t+\int_{\{h<|u-g|<h+1\}} a\left(t, x, \nabla T_{k}(u)\right) \nabla\left(T_{k}(u)-g\right) \xi \mathrm{d} x \mathrm{~d} t \\
& \leq \int_{Q} f \theta_{h}\left(T_{k}(u)-g\right) \xi \mathrm{d} x \mathrm{~d} t+\int_{\{h<|u-g|<h+1\}} G \nabla\left(T_{k}(u)-g\right) \xi \mathrm{d} x \mathrm{~d} t \\
& \quad+\int_{\Omega} R_{h}\left(T_{k}\left(u_{0}\right)\right) \xi(0, x) \mathrm{d} x+2\|\xi\|_{\infty}\left\|\lambda_{k}\right\|_{\mathcal{M}(Q)},
\end{aligned}
$$


which implies, using (2.19), (2.20), and Young's inequality,

$$
\begin{aligned}
& -\int_{Q} R_{h}\left(T_{k}(u)-g\right) \xi_{t} \mathrm{~d} x \mathrm{~d} t+\int_{\{h<|u-g|<h+1\}}\left|\nabla T_{k}(u)\right|^{p} \xi d x d t \\
& \leq \int_{Q} f \theta_{h}\left(T_{k}(u)-g\right) \xi \mathrm{d} x \mathrm{~d} t+C \int_{\{h<|u-g|<h+1\}}\left(|G|^{p^{\prime}}+|g|^{p}+|b|^{p^{\prime}}\right) \xi \mathrm{d} x \mathrm{~d} t \\
& \quad+\int_{\Omega} R_{h}\left(T_{k}\left(u_{0}\right)\right) \xi(0, x) \mathrm{d} x+2\|\xi\|_{\infty}\left\|\lambda_{k}\right\|_{\mathcal{M}(Q) .}
\end{aligned}
$$

Now, let $k \rightarrow \infty$, thanks to (4.2) and Fatou's lemma, we deduce

$$
\begin{aligned}
& -\int_{Q} R_{h}(u-g) \xi_{t} \mathrm{~d} x \mathrm{~d} t+\int_{\{h<|u-g|<h+1\}}|\nabla u|^{p} \xi \mathrm{d} x \mathrm{~d} t \\
& \leq \int_{Q} f \theta_{h}(u-g) \xi \mathrm{d} x \mathrm{~d} t+C \int_{\{h<|u-g|<h+1\}}\left(|G|^{p^{\prime}}+|g|^{p}+|b|^{p^{\prime}}\right) \xi \mathrm{d} x \mathrm{~d} t \\
& \quad+\int_{\Omega} R_{h}\left(u_{0}\right) \xi(0, x) \mathrm{d} x .
\end{aligned}
$$

Choosing $\xi=1-\frac{1}{\varepsilon} T_{\varepsilon}(t-\tau)^{+}$, for $\tau \in(0, T)$, and letting $\varepsilon \rightarrow 0$ lead to the estimate of $u-g$ in $L^{\infty}\left(0, T ; L^{1}(\Omega)\right)$. Similarly, the usual choice of nonincreasing $\xi_{\varepsilon} \in C_{c}^{\infty}([0, T))$ such that $\xi_{\varepsilon} \rightarrow 1$ allows to get

$$
\begin{aligned}
& \int_{\{h<|u-g|<h+1\}}|\nabla u|^{p} \mathrm{~d} x \mathrm{~d} t \\
& \quad \leq \int_{\{|u-g|>h\}}|f| \mathrm{d} x \mathrm{~d} t+C \int_{\{h<|u-g|<h+1\}}\left(|G|^{p^{\prime}}+|g|^{p}+|b|^{p^{\prime}}\right) \xi \mathrm{d} x \mathrm{~d} t+\int_{\left\{\left|u_{0}\right|>h\right\}}\left|u_{0}\right| \mathrm{d} x,
\end{aligned}
$$

which implies (4.32). Now, let $S \in W^{2, \infty}(\mathbb{R})$ such that $S^{\prime}$ has compact support and take a nonnegative $\xi \in C_{c}^{\infty}([0, T) \times \Omega)$. Using now the regularity (4.31), we can pass to the limit in (4.36) as $k \rightarrow \infty$, and thanks to (4.2), we obtain (4.33).

\section{Equation with absorption}

In this section, we turn to the study of equations with absorption. Let $h: \mathbb{R} \mapsto \mathbb{R}$ be a continuous function such that

$$
h(s) s \geq 0 \text { for every }|s| \geq L,
$$

for some $L \geq 0$, and let us consider the evolution problem

$$
\begin{cases}u_{t}-\operatorname{div}(a(t, x, \nabla u))+h(u)=\mu & \text { in } Q \\ u=u_{0} & \text { on }\{0\} \times \Omega \\ u=0 & \text { on }(0, T) \times \partial \Omega\end{cases}
$$

where $\mu \in \mathcal{M}_{0}(Q), u_{0} \in L^{1}(\Omega)$ and where $a: Q \times \mathbb{R}^{N} \rightarrow \mathbb{R}^{N}$ satisfies (2.19)(2.21). 
As far as the notion of solution of (5.2) is concerned, we follow the definitions introduced in the above section. Namely, a renormalized solution of (5.2) is a function $u$ such that $h(u) \in L^{1}(Q)$ and $u$ satisfies Definition 4.1 replacing $\mu$ with $\mu-h(u)$. Correspondingly, $u$ is a distributional solution if $h(u) \in L^{1}(Q)$ and $u$ is a distributional solution with $\mu-h(u)$ as right-hand side.

First of all, observe that a straightforward modification of Theorem 4.8 implies the following

THEOREM 5.1. Let $u_{1}, u_{2}$ be two renormalized solutions of problem (5.2) with data $\left(u_{01}, \mu_{1}\right)$ and $\left(u_{02}, \mu_{2}\right)$ respectively. Then,

$$
\begin{aligned}
& \int_{\Omega}\left(u_{1}-u_{2}\right)^{+}(\tau) \mathrm{d} x+\int_{0}^{\tau} \int_{\Omega}\left(h\left(u_{1}\right)-h\left(u_{2}\right)\right) \operatorname{sign}^{+}\left(u_{1}-u_{2}\right) \mathrm{d} x \mathrm{~d} t \\
& \leq\left\|\left(u_{01}-u_{02}\right)^{+}\right\|_{L^{1}(\Omega)}+\left\|\left(\mu_{1}-\mu_{2}\right)^{+}\right\|_{\mathcal{M}(Q)}
\end{aligned}
$$

for almost every $\tau \in(0, T)$.

When $h$ is monotone, the above $L^{1}$-contraction principle plays a crucial role in such type of problems. We are now able to prove our main result concerning (5.2).

THEOREM 5.2. Let $\mu \in \mathcal{M}_{0}(Q)$ and $u_{0} \in L^{1}(\Omega)$ and let $h$ be a continuous function satisfying (5.1). Then, problem (5.2) admits a renormalized solution (in particular, a distributional solution). If, in addition, $h$ is nondecreasing, then the renormalized solution is unique.

Proof. As in the proof of Proposition 4.7, we take $\mu_{n}=\mu * \rho_{n}$, where $\left(\rho_{n}\right)$ is a sequence of mollifiers. We then consider the solutions $u_{n}$ of

$$
\begin{cases}\left(u_{n}\right)_{t}-\operatorname{div}\left(a\left(t, x, \nabla u_{n}\right)\right)+h\left(u_{n}\right)=\mu_{n} & \text { in } Q, \\ u_{n}=u_{0 n} & \text { on }\{0\} \times \Omega, \\ u_{n}=0 & \text { on }(0, T) \times \partial \Omega .\end{cases}
$$

Since $\left\|\mu_{n}\right\|_{\mathcal{M}(Q)} \leq\|\mu\|_{\mathcal{M}(Q)}$, using assumption (5.1), one easily gets that $\left(h\left(u_{n}\right)\right)$ is bounded in $L^{1}(Q)$ and $\left\|h\left(u_{n}\right)\right\|_{L^{1}(Q)} \leq\|\mu\|_{\mathcal{M}(Q)}$. Hence, the sequence $\left(u_{n}\right)$ satisfies the estimates of Proposition 4.6 and the compactness properties. In particular, there exists $u \in L^{1}(Q)$ such that (4.17) holds up to a subsequence. Moreover, Theorem 1.2 implies that

$$
\lim _{k \rightarrow \infty} \sup _{n} \operatorname{cap}_{p}\left\{\left|u_{n}\right|>k\right\}=0 .
$$

Multiplying the equation in (5.4) by $T_{1}\left(u_{n}-T_{k}\left(u_{n}\right)\right)$, we obtain

$$
\int_{\left\{\left|u_{n}\right|>k+1\right\}}\left|h\left(u_{n}\right)\right| \leq \int_{\left\{\left|u_{n}\right|>k\right\}}\left|\mu_{n}\right| .
$$

By Proposition 3.3, the sequence $\left(\mu_{n}\right)$ is equidiffuse, so that we get

$$
\lim _{k \rightarrow \infty} \sup _{n} \int_{\left\{\left|u_{n}\right|>k+1\right\}}\left|h\left(u_{n}\right)\right|=0
$$


We now prove the equi-integrability of the sequence $\left(h\left(u_{n}\right)\right)$. Indeed, since for any subset $E \subset Q$, we have

$$
\sup _{n} \int_{E}\left|h\left(u_{n}\right)\right| \leq \sup _{n} \int_{E}\left|h\left(T_{k+1}\left(u_{n}\right)\right)\right|+\sup _{n} \int_{\left\{\left|u_{n}\right|>k+1\right\}}\left|h\left(u_{n}\right)\right|,
$$

and since, for fixed $k$, the sequence $\left(h\left(T_{k+1}\left(u_{n}\right)\right)\right.$ is equi-integrable, we deduce that

$$
\lim _{|E| \rightarrow 0} \sup _{n} \int_{E}\left|h\left(u_{n}\right)\right| \leq \sup _{n} \int_{\left\{\left|u_{n}\right|>k+1\right\}}\left|h\left(u_{n}\right)\right| .
$$

Hence, letting $k \rightarrow \infty$, we get the equi-integrability of $\left(h\left(u_{n}\right)\right)$. Since $\left(h\left(u_{n}\right)\right)$ converges pointwise to $h(u)$, by Vitali's theorem,

$$
h\left(u_{n}\right) \rightarrow h(u) \text { strongly in } L^{1}(Q) .
$$

We can apply now Proposition 4.7 to deduce that $u$ is a renormalized solution of (5.2). When $h$ is nondecreasing, we obtain uniqueness of the renormalized solution from Theorem 5.1.

In the case where $h$ is nondecreasing, the existence of a solution can also be proved in a slightly different way, which consists in proving first the result for a dense subset of measures $\mu$, then using the $L^{1}$-contraction principle (Theorem 5.1) to obtain the result for any diffuse measure $\mu$. We can take for instance the subset of measures satisfying the decomposition (1.5) with $g \in L^{\infty}(Q)$; this set is dense in view of Theorem 1.1. For such measures, the existence of solutions of (5.2) can be proved in the lines of the elliptic case.

\section{Extension to the nonmonotone case}

The approach developed in this paper is not limited to the case that the divergence form operator is monotone. Let for example $a: Q \times \mathbb{R} \times \mathbb{R}^{N} \rightarrow \mathbb{R}^{N}$ be a Carathéodory function (i.e., $a(\cdot, \cdot, s, \xi)$ is measurable on $Q$ for every $(s, \xi)$ in $\mathbb{R} \times \mathbb{R}^{N}$, and $a(t, x, \cdot, \cdot)$ is continuous on $\mathbb{R} \times \mathbb{R}^{N}$ for almost every $(t, x)$ in $Q$ ) such that the following holds:

$$
\begin{aligned}
& a(t, x, s, \xi) \cdot \xi \geq \alpha|\xi|^{p}, \\
& |a(t, x, s, \xi)| \leq \beta\left[b(t, x)+|s|^{p-1}+|\xi|^{p-1}\right], \\
& {[a(t, x, s, \xi)-a(t, x, s, \eta)] \cdot(\xi-\eta)>0,}
\end{aligned}
$$

for almost every $(t, x)$ in $Q$, for every $s \in \mathbb{R}$, and for every $\xi, \eta$ in $\mathbb{R}^{N}$, with $\xi \neq \eta$, where, as before, $p>1, \alpha$ and $\beta$ are two positive constants, and $b$ is a nonnegative function in $L^{p^{\prime}}(Q)$. From (6.1), we can deduce that $a(x, t, s, 0)=0$ for any $s \in \mathbb{R}$ and a.e. $(t, x) \in Q$. Consider the problem

$$
\begin{cases}u_{t}-\operatorname{div}(a(t, x, u, \nabla u))+h(u)=\mu & \text { in } Q, \\ u=u_{0} & \text { on }\{0\} \times \Omega, \\ u=0 & \text { on }(0, T) \times \partial \Omega,\end{cases}
$$


where $\mu$ is a diffuse measure, $u_{0} \in L^{1}(\Omega)$, and $h$ satisfies (5.1). The method developed to find existence of solutions relies on the possibility to find capacitary estimates. The proof we have given of such estimates in Theorem 1.2 (and Theorem 2.2) used the monotone character of the second order term, but we can generalize these estimates in the following

THEOREM 6.1. Assume that (6.1)-(6.3) hold. Given $u_{0} \in L^{2}(\Omega)$ and $\mu \in$ $\mathcal{M}(Q) \cap L^{p^{\prime}}\left(0, T ; W^{-1, p^{\prime}}(\Omega)\right)$, let $u \in W$ be a (weak) solution of

$$
\begin{cases}u_{t}-\operatorname{div}(a(t, x, u, \nabla u))=\mu & \text { in } Q \\ u=u_{0} & \text { on }\{0\} \times \Omega, \\ u=0 & \text { on }(0, T) \times \partial \Omega .\end{cases}
$$

Then,

$$
\operatorname{cap}_{p}(\{|u|>k\}) \leq C \max \left\{\frac{1}{k^{\frac{1}{p}}}, \frac{1}{k^{\frac{1}{p^{\prime}}}}\right\} \quad \forall k \geq 1
$$

where $C>0$ is a constant depending on $\|\mu\|_{\mathcal{M}(Q)},\left\|u_{0}\right\|_{L^{1}(\Omega)},\|b\|_{L^{p^{\prime}}(Q)}, \alpha, \beta, p$ and $\Omega$.

Proof. The strategy is the same of the proof of Theorem 1.2 so we only sketch the main technical changes. Let us define the auxiliary function $\tilde{a}(t, x, \xi) \equiv a\left(t, x, u^{+}, \xi\right)$. Note that, due to (6.2),

$$
|\tilde{a}(t, x, \xi)| \leq \beta\left[b(t, x)+\left(u^{+}\right)^{p-1}+|\xi|^{p-1}\right]
$$

and since $u \in L^{p}\left(0, T ; W_{0}^{1, p}(\Omega)\right)$, we have that $\tilde{a}(t, x, \xi)$ satisfies (2.20) (and clearly also (2.19) and (2.21)). Since $\mu$ is diffuse then $\mu^{+}$is diffuse as well; we then consider the unique renormalized solution $v$ of the following problem

$$
\begin{cases}v_{t}-\operatorname{div}(\tilde{a}(t, x, \nabla v))=\mu^{+} & \text {in } Q, \\ v=u_{0}^{+} & \text {on }\{0\} \times \Omega, \\ v=0 & \text { on }(0, T) \times \partial \Omega .\end{cases}
$$

It follows by Theorem 4.8 (see Remark 4.5) that $u^{+} \leq v$. Now, as in Proposition 4.8, we can prove that

$$
T_{k}(v)_{t}-\operatorname{div}\left(\tilde{a}\left(t, x, \nabla T_{k}(v)\right)\right) \geq 0 \text { in } \mathcal{D}^{\prime}(Q)
$$

Without loss of generality, assume that $T_{k}(v(T))$ is well defined (otherwise use a sequence $t_{n} \uparrow T$ such that $T_{k}\left(v\left(t_{n}\right)\right)$ is so). We define the function $z \in W$ as the solution of

$$
\begin{cases}-z_{t}-\operatorname{div}(\tilde{a}(t, x, \nabla z))=-2 \operatorname{div}\left(\tilde{a}\left(t, x, \nabla T_{k}(v)\right)\right) & \text { in } Q, \\ z=T_{k}(v) & \text { on }\{T\} \times \Omega, \\ z=0 & \text { on }(0, T) \times \partial \Omega,\end{cases}
$$


and we apply a comparison $\operatorname{argument}(\operatorname{since}-\operatorname{div}(\tilde{a}(t, x, \nabla z))$ is monotone $)$ to deduce

$$
z \geq T_{k}(v) \geq T_{k}\left(u^{+}\right) \quad \text { a.e. in } Q .
$$

Now, $T_{k}(v)$ satisfies the usual estimates (2.6) (e.g., by Proposition 4.5) and since

$$
\begin{aligned}
\left|\tilde{a}\left(t, x, \nabla T_{k}(v)\right)\right|^{p^{\prime}} & \leq C\left[b(t, x)^{p^{\prime}}+\left(u^{+}\right)^{p}+\left|\nabla T_{k}(v)\right|^{p}\right] \chi_{\{v<k\}} \\
& \leq C\left[b(t, x)^{p^{\prime}}+\left(T_{k}(v)\right)^{p}+\left|\nabla T_{k}(v)\right|^{p}\right],
\end{aligned}
$$

using Poincaré inequality, we have

$$
\left\|\tilde{a}\left(t, x, \nabla T_{k}(v)\right)\right\|_{L^{p^{\prime}}(Q)} \leq C\left[\|b\|_{L^{p^{\prime}}(Q)}+\left\|\nabla T_{k}(v)\right\|_{L^{p}(Q)}^{p-1}\right] \leq C\left(1+k^{\frac{p-1}{p}}\right) .
$$

Therefore, $z$ satisfies the estimate in $W$ as in Step 2 of Theorem 1.2, and we conclude that

$$
\operatorname{cap}_{p}(\{u>k\}) \leq C \max \left\{\frac{1}{k^{\frac{1}{p}}}, \frac{1}{k^{\frac{1}{p^{\prime}}}}\right\} \quad \forall k \geq 1 .
$$

The estimate for $\{u<-k\}$ follows in the same way using $\mu^{-}$and $u^{-}$.

Finally, once we have the capacitary estimates in hand, we can follow the proof of Theorem 5.2 and, through approximation, we can find the existence of at least one renormalized (in particular, weak) solution of (6.4).

\section{REFERENCES}

[1] P. BARAS AND M. PIERRE, Problèmes paraboliques semi-linéaires avec données mesures, Applicable Anal. 18 (1984), 111-149.

[2] P. Bénilan, L. Boccardo, T. Galloukt, R. Gariepy, M. Pierre and J. L. Vazquez, An $L^{1}$-theory of existence and uniqueness of nonlinear elliptic equations, Ann. Scuola Norm. Sup. Pisa Cl. Sci. (4) 22 (1995), 241-273.

[3] P. BÉNILAN, J. CARRILlO AND P. WitTBOLD, Renormalized entropy solutions of scalar conservation laws, Ann. Scuola Norm. Sup. Pisa Cl. Sci. (4) 29 (2000), 313-327.

[4] D. BLANCHARD AND F. MURAT, Renormalized solutions of nonlinear parabolic problems with $L^{1}$ data: existence and uniqueness, Proc. Royal Soc. Edinburgh Sect. A 127 (1997), 1137-1152.

[5] D. BLANCHARD AND A. PORRETTA, Stefan problems with nonlinear diffusion and convection, J. Diff. Eq. 210 (2005), 383-428.

[6] L. Boccardo, A. Dall'Aglio, T. GallouEt And L. Orsina, Nonlinear parabolic equations with measure data, J. Funct. Anal. 147 (1997), 237-258.

[7] L. BOCCARDO AND T. GALLOUET, Nonlinear elliptic and parabolic equations involving measure data, J. Funct. Anal. 87 (1989), 149-169.

[8] L. Boccardo, T. GAllouEt AND L. ORsina, Existence and uniqueness of entropy solutions for nonlinear elliptic equations with measure data, Ann. Inst. H. Poincaré Anal. Non Linéaire 13 (1996), 539-551.

[9] H. BRezIS, M. MARCUS AND A. C. PONCE, Nonlinear elliptic equations with measures revisited. In: Mathematical Aspects of Nonlinear Dispersive Equations (J. Bourgain, C. Kenig, and S. Klainerman, eds.), Annals of Mathematics Studies, 163, Princeton University Press, Princeton, NJ, 2007, pp. 55-110.

[10] H. BREZIS AND A. C. PONCE, Reduced measures for obstacle problems, Adv. Diff. Eq. 10 (2005), $1201-1234$. 
[11] G. DAL MASO AND A. MALUSA, Some properties of reachable solutions of nonlinear elliptic equations with measure data, Ann. Scuola Norm. Sup. Pisa Cl. Sci. 25 (1997), 375-396.

[12] G. DAl Maso, F. Murat, L. Orsina AND A. PRIGnet, Renormalized solutions of elliptic equations with general measure data, Ann. Scuola Norm. Sup. Pisa Cl. Sci. 28 (1999), 741-808.

[13] J. DRoniou, A. PorretTA AND A. PRIGNET, Parabolic capacity and soft measures for nonlinear equations, Potential Anal. 19 (2003), 99-161.

[14] J. DRONIOU AND A. PRIGNET, Equivalence between entropy and renormalized solutions for parabolic equations with smooth measure data, NoDEA Nonlinear Diff. Eq. Appl. 14 (2007), 181-205.

[15] L. C. EVANS AND R. F. GARIEPY, Measure Theory and fine properties of functions, Studies in Advanced Mathematics, CRC Press, Boca Raton, FL, 1992.

[16] T. Galloutet And J. M. Morel, Resolution of a semilinear equation in $L^{1}$, Proc. Roy. Soc. Edinburgh Sect. A 96 (1984), 275-288.

[17] J.- L. LIONS, Quelques méthodes de résolution des problèmes aux limites non linéaires, Paris, Dunod, 1969.

[18] M. MARCUS AND A. C. PONCE, Reduced limits for nonlinear equations with measures, J. Funct. Anal. 258 (2010), 2316-2372.

[19] F. PETITTA, Renormalized solutions of nonlinear parabolic equations with general measure data, Ann. Mat. Pura Appl. (4) 187 (2008), 563-604.

[20] F. Petitta, A. C. POnce AND A. Porretta, Approximation of diffuse measures for parabolic capacities, C. R. Acad. Sci. Paris 346 (2008), 161-166.

[21] M. PIERRE, Parabolic capacity and Sobolev spaces, Siam J. Math. Anal. 14 (1983), 522-533.

[22] A. PORRETTA, Existence results for nonlinear parabolic equations via strong convergence of truncations, Ann. Mat. Pura Appl. (4) 177 (1999), 143-172.

\section{F. Petitta}

Universitat de Valencia,

Departamento de Análisis Matemático,

C/Dr. Moliner 50,

46100 Burjassot, Valencia, Spain

E-mail: francesco.petitta@uv.es

A. C. Ponce

Université catholique de Louvain,

Institut de Recherche en Mathématique et Physique,

Chemin du Cyclotron 2,

1348 Louvain-la-Neuve, Belgium

E-mail: augusto.ponce@uclouvain.be

A. Porretta

Università di Roma Tor Vergata,

Dipartimento di Matematica,

Via della ricerca scientifica 1 ,

00133 Rome, Italy

E-mail:porretta@mat.uniroma2.it 